\title{
Can thermodynamics guide us to make better solar cells ?
}

\author{
Tom Markvart \\ Engineering Sciences, University of Southampton, Southampton SO17 1BJ, UK, and \\ Centre for Advanced Photovoltaics, Czech Technical University, 16636 Prague 6, Czech Republic
}

\begin{abstract}
Thermodynamics has provided a powerful tool to study radiation and its conversion into useful work. Starting from the so-called Shockley's paradox, this paper discusses the thermodynamic view of fundamental losses to photovoltaic conversion, and how thermodynamics enters the charge carrier transport in semiconductors and heat exchange processes at p-n junctions. Turning to photon flows, considerations based on detailed balance and reciprocity provide a comprehensive picture of the voltage produced by the solar cell in the presence of nonradiative recombination. We shall use these tools to examine several topics under recent discussion, including photon recycling and hot carrier conversion based on thermoelectricity.
\end{abstract}

\section{Introduction}

Ever since acting as a springboard for the development of quantum theory, the thermodynamics of radiation has provided a fertile environment for the discussion of new ideas in solar energy conversion. This has never been more so than since the birth of modern photovoltaics some seventy years ago. When combined with powerful statistical tools such as various forms of detailed balance and reciprocity, thermodynamics has more recently re-emerged as a useful tool for testing the viability of new proposals, broadening the understanding of some - even quite traditional - concepts, and suggesting new mechanisms. Divided, broadly speaking, into three parts which focus on the thermodynamics of light, charge carriers transport in semiconductors and the principles of detailed balance and reciprocity which link the two, this paper discusses the fundamental losses in PV conversion and the heat exchange which accompanies carrier transport in a p-n junction, Classical and more recent reciprocity theorems are used to discuss the role of nonradiative recombination and photon recycling. The paper concludes by taking a look how the junction thermodynamics can be applied to form new ideas about hot carrier solar cells based on thermoelectricity.

\section{Where is thermodynamics in photovoltaics?}

\section{(a) From Planck to Shockley and the chemical potential of light}

The application of thermodynamics to light (or, more generally, electromagnetic radiation) has a famous history. Building on earlier achievements of a number of scientists, notably Kirchhoff and Wien, investigating thermal (or black body) radiation led Planck, at the turn of the $20^{\text {th }}$ century, to his celebrated radiation law ${ }^{1}$

$\phi_{v}(T)=2\left(\frac{v}{c}\right)^{2} \frac{1}{e^{h v / k_{B} T}-1}$

Planck's formula is expressed here in terms of the number of photons $\phi_{v}$ per unit frequency $v$ passing through a unit area per unit time per unit solid angle in vacuum, $c$ is the speed of light, $h$ is the Planck constant and $k_{B}$ is the Boltzmann constant, Equation (1) describes electromagnetic radiation in thermal equilibrium with matter at temperature $T$. In fact, two forms of equilibrium are present

\footnotetext{
${ }^{1}$ At the time of his discovery Planck was not aware of the existence of photons, and his radiation law describes radiant power rather than photon flux. With application to photovoltaics, however, it is more convenient to express Planck's law in the form (1).
} 
here: it relies on the concept of isotropic "radiation gas" to which Planck's formula (1) ascribes a distribution in the frequency (or wavelength or photon energy) space. We shall see that both aspects are relevant for applications to photovoltaics.

The spectrum of light arriving from the Sun has a close resemblance to the black body spectrum at a temperature $T_{S}$ close to $6000 \mathrm{~K}$. Being able to assign temperature to solar radiation, it is tempting to apply another classical results - Carnot's law for the efficiency of an ideal heat engine - to solar cells, with the seemingly obvious result

$$
\eta_{P V}=1-\frac{T_{o}}{T_{S}}
$$

where $T_{o}$ is the solar cell temperature, assumed equal to the ambient temperature. Even though - as we shall see - Carnot efficiency does play an important role in photovoltaics - care is required before we apply Eq. (2) to solar cells. The Carnot efficiency is the ratio of energies: the work carried out divided by the heat absorbed, in one cycle. For reversible operation, the Carnot engine has to turn very slowly, each cycle taking effectively an infinite time interval to complete. The rate at which the engine deliver energy, per unit time (in other words, power) is therefore zero. The efficiency of a solar cell, in contrast, is a power efficiency: the ratio of power output to the radiant power absorbed. A different angle is therefore required to find a suitable thermodynamic parallel. Notwithstanding, this argument provides a suitable direction, to search for a suitable energy characteristics of a solar cell to which a thermodynamic argument could be applied.

Planck's radiation law holds for thermal radiation, emitted by a heated (black) body in thermal equilibrium. To apply this concept to solar cells, a generalisation is needed for luminescent sources excited by an external perturbation such as illumination (solar cells) or applied voltage (light emitting diodes,
LEDs). In operation, these devices - which emit so-called cold light - are not in complete equilibrium but in a steady state: whilst in equilibrium at a given temperature, they are characterised by well defined numbers of photons in the emitted beam - for example, the photon fluxes absorbed and emitted by an ideal solar cell are equal. In contrast with the black body radiation, thermodynamics now implies that it is meaningful to assign the emitted light a chemical potential, to be denoted by $\mu^{1}{ }^{2}$, transforming Planck's law to

$\phi_{\nu}(T, \mu)=2\left(\frac{v}{c}\right)^{2} \frac{1}{e^{(h v-\mu) / k_{B} T}-1}$

Equation (3) was first applied to solar cells by Würfel ${ }^{3}$.

If absorbed fully by a semiconductor, the incident photon flux (1) can be equated to the emitted flux described with the use of (3) and used to determine the chemical potential ( $\mu_{o}$, say) of the emitted photons. Applying the reasoning of thermodynamics one can then argue that the same chemical potential should apply to the emitting electron-hole pairs which can produce work $q V$ at a semiconductor junction, where $V$ is the voltage generated by the solar cell and $q$ is the elementary charge. This is the essence of a well-known detailedbalance result for the efficiency of an ideal solar cell, obtained in a more intuitive manner by Shockley and Queisser in $1961{ }^{4}$

Having stressed the thermodynamic nature of this result, we can pursue this line of thought further, and express the average energy $u$ per photon as a sum the chemical potential $\mu$ and a term which can be described as heat:

$u=\mu+T s$

where $u$ and $s$ are the energy and entropy of adding a photon to a beam or volume of radiation. Equation (4) is a rigorous result in both the equilibrium and nonequilibrium thermodynamics, although there is a slight difference in interpretation of $u$ and $s$ in the 
two cases. ${ }^{5,19,53}$. It is particularly simple in the case of photons which do not intereact with each other, and the energy $u$ and entropy $s$ are simple derivatives at constant volume (or constant étendue, in the case of a light beam). ${ }^{10,11}$ Equation (4) represents a key result for the understanding of the operation of a solar cell in thermodynamic terms as it separates from the photon energy $u$ a part (the chemical potential) which can be usefully extracted to carry out work or generate voltage in an external circuit. The second term - which introduces the notion of entropy - is equal to the heat that needs to be exchanged with a reservoir at the same temperature as the source of photons when a photon is emitted or absorbed.

Equation (4) can be illustrated on the example of the Shockley paradox, reported by Al Rose in $1960 .{ }^{6}$ Paraphrasing the details, the paradox can be summarised as follows. A forward bias of $0.1 \mathrm{~V}$ is applied to a solar cell made from a semiconductor with bandgap $1 \mathrm{eV}$, operating as an LED. The LED radiates a flux of photons with energy slightly above $1 \mathrm{eV}$ which illuminates a second, identical, solar cell, giving rise to a current $I$ of $1 \mathrm{~V}$ electrons. The second solar cell can be connected to the first to produce a self-sustaining source of light.

Equation (4) allows a simple resolution of this paradox. The first solar cell / LED emits a beam of light containing photons with energy just above $1 \mathrm{eV}$ but a chemical potential $\mu_{\text {LED }}$ ( $=q V_{L E D}$ ) of only $0.1 \mathrm{eV}$. By virtue of (4), the difference between these two is heat. The second solar cell converts this light into voltage $V_{P V}$; since the conversion takes place at constant temperature, $q V_{P V}$ can, at most, be equal to $\mu_{L E D}$. For useful operation, the solar cell has to generated a finite current which, as we shall see below, introduces losses by irreversibility and therefore $V_{P V}<V_{L E D}$. The key point of this argument is that an LED excited by a low voltage emits a low-intensity beam of photons which carry a high entropy content and which cannot be converted to voltage in an isothermal process.

Considerations of the entropy carried by photons become particularly relevant for solar radiation where, using the parallel with a black body, the chemical potential is equal to zero. Since the heat carried by solar photons cannot be exploited externally without bringing in a heat bath at a different temperature, a solar cell - or any other device that converts solar radiation to useful work - must work as a heat engine, subject to the laws of thermodynamics (Fig. 1).

Accordingly, we can immediately write down an equation for the energy $q V$ produced by a photon in a form of an incident photon energy $u_{S}$ converted with the Carnot efficiency, less any losses due to the (irreversible) entropy $\sigma_{i}$ per photon generated in the conversion process: ${ }^{7,8,9}$

$q V=\mu_{o}=\left(1-\frac{T_{o}}{T_{S}}\right) u_{S}-T_{o} \sigma_{i}$

The irreversible entropy generation $\sigma_{i}$ per photon, alongside the Carnot efficiency, make it possible to quantify the fundamental contribution to voltage losses in an ideal solar cell. ${ }^{7,10}$ These losses, as contributions to $\sigma_{i}$, can be conveniently discussed alongside the principal steps of the conversion process.

The parameters of an ideal solar cell are set by the photon balance between absorption and emission less any photons that are extracted for conversion to current. At open circuit, photons absorbed at the temperature $T_{S}$ of solar radiation are cooled to the ambient temperature $T_{o}$ and emitted from the cell. The cooling process (often described through the thermalization of electron-hole pairs that emit these photons) generates entropy $\sigma_{\text {cool }}$ and incurs losses through the rejection of heat and the change of photon entropy $s_{S}$ at temperature $T_{S}$ to $s_{o}$ at $T_{o}$ : 
$q \Delta V_{\text {cool }}=T_{o} \sigma_{\text {cool }}=\left(u_{S}-u_{o}\right)-T_{o}\left(s_{S}-s_{o}\right)$

where $u_{o}$ is photon energy at temperature $T_{o}$ The corresponding voltage loss $\Delta V_{\text {cool }}$ can then be readily determined as described in refs. 7 , 10 and 11 . This immediately leads to an idea how solar cell efficiency can be increased: a hot carrier solar cell where the irreversible cooling by thermalization is replaced by reversible cooling in a Carnot-like engine, without entropy generation, thus gaining voltage $\Delta V_{\text {cool }}$. Figure 2 gives this voltage gain for a more general model where the reversible energy extraction is preceded by irreversible thermalization to a temperature $T_{h c}\left(\leq T_{S}\right)$.

The second loss is due to the expansion in the size of the beam. ${ }^{11}$ Solar radiation arrives at the Earth within a solid angle $\omega_{s}=6.85 \times 10^{-5}$ sterad but the solar cell emits the low-temperature radiation into a full hemisphere. The expansion of the beam in the conversion process is characterised by the ratio of the two étendues. The étendue for photons in a beam is analogous to the volume for the ideal gas. For a solar cell operating under one-sun illumination, étendue expansion in the conversion process $\sigma_{\text {exp }}$ is equal to $k_{B} \ln \left(\pi / \omega_{s}\right)\left(\omega_{s}=\right.$ $6.85 \times 10^{-5}$ sterad is the solid angle subtended by the Sun) reducing the open-circuit voltage by $277 \mathrm{mV}$ at 300K. An ideal hot-carrier solar cell under one-sun illumination ${ }^{2}$ (in other words, with only étendue expansion loss $\sigma_{\text {exp }}$ but no losses $\sigma_{\text {cool }}$ by thermalization) would produce an open-circuit voltage of $1.607 \mathrm{~V}$. For a silicon like cell, this translates to an efficiency of $58 \%$.

The third loss occurs if the solar cell produces a finite current: this is the reason why Carnot efficiency (here applied to the output energy $q V$ ) cannot be reached by a dynamical converter which produces power; A finite rate of turnover (equivalent to current extraction from the cell) generates entropy which reduces

\footnotetext{
${ }^{2}$ In other words, photon flux with black-body spectrum (1) with $T=6000 \mathrm{~K}$, limited to a solid angle of $\omega_{s}$.
}

the voltage from the open circuit value. Viewed in a different way, a solar cell producing finite current emits fewer photons than it absorbs. The emitted beam therefore has a higher entropy per photon than the incident beam - the difference equal to the entropy generated in the conversion process.

The thermodynamic description of solar cell operation therefore considers energy as the key parameter, leading to a V-I characteristic shown in Fig. 3 together with the losses described by the various terms in Eq. (5). This approach - similar to electrochemistry - can be contrasted with the traditional treatment which start from the transport equations for carrier transport in the solar cell, which are solved for current, producing the usual solar cell equation.

\section{(b) Thermodynamics of carrier transport in semiconductors}

Carrier transport in semiconductors is a highly developed and mature field (see, for example, ${ }^{12,13,14,15}$ ). Yet, when applied to devices such as solar cells, debates about fundamental issues persist. Two points, in particular, repeatedly occur in the scientific literature. The balance between drift and diffusion currents, and the built-in electric field which appears at a semiconductor junction: is it important / relevant for the solar cell operation ${ }^{16,17}$ ? Selective contacts have been suggested, either to separate charges (as a replacement of the electric field above), or energy selective contacts, as part of the Ross and Nozik model of hot carrier solar cells. Although some attempts at a theoretical description have been made ${ }^{18}$ details required for in-depth modelling appear to be limited.

It might appear that thermodynamics only adds to this confusion. Take the Ohm's law. The differential form of Ohm's law for current density $J$ can be written as $J=-\sigma \nabla \phi$, where $\sigma$ is the conductivity and the gradient of the electrostatic potential $\phi$ replaces the voltage 
difference. In contrast, in semiconductors - in keeping with the broader field of nonequilibrium thermodynamics ${ }^{19}$ - we usually find, for example, for electrons,

$$
J=(\sigma / q) \nabla \mu
$$

where the (electro-)chemical potential $\mu$ of the charge carriers stands in for the Fermi energy $E_{F}$. How can these two versions be reconciled?

The evident answer, of course, is that the traditional form of Ohm's law includes only drift transport whereas the form containing the chemical potential includes also carrier diffusion. Following this argument further we substitute expression (4) into (7) to obtain,

$$
J=(\sigma / q) \nabla u-(\sigma / q) T \nabla s=-\sigma \nabla \phi+D \nabla n
$$

where we used the results for electrons

$$
u=E_{c}-q \phi+u_{t} ; \quad s=k_{B}\left\{\ln \left(N_{c} / n\right)+u_{t} / T\right\}
$$

where $N_{c}$ is the effective density of states in the conduction band, $u_{t}$ is the thermal energy of an electron that, for an ideal gas, can be equated to (3/2) $k_{B} T$ and the diffusion constant $D$ was inserted to replace the conductivity in the last equation with the help of Einstein's relation $\sigma=q^{2} \mathrm{Dn} /\left(k_{B} T\right)$ to obtain an equation for the electron current density in the usual form. A corresponding equations can be written down for holes.

Strictly speaking, the use of (4) for charge carriers in semiconductors away from equilibrium should contain corrections to the energy $u_{t}$ and entropy $s$ which describe their contribution to current (see p. 26 of ref. 20). Nevertheless, the use of equilibrium values remains a good approximation. ${ }^{21}$ We shall see in Sec. 3 that it parallels Lord Kelvin's treatment of thermoelectricity, ${ }^{22}$ and is easy to interpret and visualise. Pursuing further the language of nonequilibrium thermodynamics, equation (7) depicts the two driving forces responsible for electrical current: the gradient of electron energy (or, in this case, the electrostatic potential), and the gradient of entropy which drives the diffusion current.

Let us now apply this philosophy to a p-n junction. At open circuit $(J=0)$, Eq. (8) gives $\mu=$ constant across the junction, ${ }^{3}$ and the change of the electrostatic energy (due to the built-in voltage $V_{b i}$ ) is equal to the change of entropy per carrier. Using the standard result

$$
q V_{b i}=k_{B} T \ln \left(\frac{n_{\text {on }}}{n_{o p}}\right)=k_{B} T \ln \left(\frac{v_{p}}{v_{n}}\right)=\int_{v_{n}}^{v_{p}} p d v
$$

In the second equation on the right hand side of (10) we introduced the volume per carrier $v$ $=1 / n_{o}$ on the two sides of the junction, and the last equation was transformed with the use of the ideal gas law $p v=k_{B} T$ to introduce the pressure $p$ and work per carrier $p d v$. In other words, the change of electron energy across the junction due to the built in field is equal to the work done on or by the electron, by compression or expansion on the n-side $\left(v_{n}\right)$ to the p-side $\left(v_{p}\right)$. This "internal work" which cannot be extracted in an isothermal process for external use - corresponds to a change of entropy, indicating that heat is absorbed or rejected. The important point to note that this is a reversible isothermal process where no external work (or voltage) is produced but heat is exchanged with the reservoir to produce internal work on, or by, the electric field. We shall see in Sec. 3 this isothermal step can be expanded into a full thermodynamic cycle to convert the carrier heat into useful work in a hot carrier solar cell.

\footnotetext{
${ }^{3}$ To a good level of accuracy, this result holds even away from the open circuit by virtue of the fact that the electron transit time across the junction is very short and the excess carrier concentration injected into the junction by the generation current is therefore very small. It is then a small step to show the $\mu=$ constant across the junction holds more generally under the conditions of operation of solar cells.
} 


\section{Detailed balance and reciprocity: voltage and photon recycling}

The voltage generated by solar cells is determined by the carrier concentrations at the junction. These - essentially static - quantities are in turn determined by the fluxes of photons discussed in Sec. 1. A direct link between these fluxes and voltage is furnished by various principles of detailed balance.

We have already met the detailed balance considered by Shockley and Queisser to model the operation of an ideal solar cell. In the language of thermodynamics, the principle of detailed balance has a more general meaning: the equality of two opposing flows in thermal equilibrium. In chemistry, for example, the forward and reverse reaction rates are equal in equilibrium. The rates can then be applied to situations away from equilibrium - in our case, to various processes which occur in the absorption and emission of light.

The two principal relations between absorption and emission of radiation by matter are summarised in Fig. 4a. ${ }^{23}$ The first relationship of this kind was formulated by Kirchhoff who postulated that the ratio of the rate at which energy (or photons, in our terminology) is emitted by a unit area to the absorptivity $a$ is equal to a universal function of the temperature of the substance and the wavelength. This universal function was discovered by Planck (see Eq. (1)) who noted that radiation can only be absorbed or generated in a volume element of a body rather than on the surface. ${ }^{24}$ This balance can be expressed in terms of the absorption coefficient $\alpha_{v}$ and the coefficient of emission $\varepsilon_{v}$. Einstein later extended Planck's photon balance to describe transitions between individual quantum states. discovering at the same time, stimulated emission. Einstein's detailed balance was later generalised to molecules by Kennard ${ }^{25}$ and Stepanov. ${ }^{26}$, and to semiconductors by van Roosbroeck and Shockley. ${ }^{27}$
Further balance relations were developed more recently to serve the needs of the modern optoelectronic devices such as LEDs, lasers and solar cells, and to accommodate phenomena which take place in these devices. These include the reabsorption of the internally emitted light (or photon recycling). Photon recycling has been considered for some time (see, for example, ${ }^{28}$ ), principally as a means of extending the diffusion lengths and radiative lifetimes. ${ }^{29}$ The concept has recently reemerged with the suggestion that it may help increase the voltage in the latest generation of solar cells with a high radiative efficiency.

Although not considered explicitly in the traditional framework, photon recycling already exists as a link between Kirchhoff's laws describing emission from the surface and Planck's volume emission. It can be quantified on this basis by equating the rate of photon emission from the surface to the rate of internal emission multiplied by the probability that a photon is not reabsorbed $(1-r)$ (Appendix A; see also ref. 23):

$a_{o}=\ell_{\text {opt }}\langle\alpha\rangle_{o}(1-r)$

where $r$ is the average probability that an internally emitted photon is reabsorbed, $\langle\alpha\rangle_{0}$, and $a_{o}$ are the average absorption coefficient and absorptivity for the emitted light, and, for a planar structure of thickness $d, \ell_{\text {opt }}=4 n_{\text {ref }}^{2} d$ where $n_{r e f}^{2}$ is the refractive index of the medium

The length $\ell_{\text {opt }}$ is the well known Yablonovitch result for the (maximum) path length of photons in a medium with perfect light trapping. Although usually implemented by using surface texture, light trapping in a more general sense can be understood to mean any mechanism that produces isotropic radiation in other words, Planck's radiation gas, in a directional equilibrium.

The average absorption coefficient $\langle\alpha\rangle_{0}$ is of the order of $\sim 1 \mathrm{~mm}^{-1}$ in indirect gap 
materials such as silicon, and $\sim 1 \mu \mathrm{m}^{-1}$ for direct gap semiconductors. Its inverse is a measure of the thickness of a shell near the surface (the "photosphere") from which light is emitted to the exterior of the material; photons emitted deeper in the materials will be reabsorbed (Fig. 5). Applying Eq. (11) for optically thick structures with $a_{o} \cong 1$ (see Appendix A) then gives the reabsorption probability $r \cong 1-\left\{\ell_{\text {opt }}\langle\alpha\rangle_{0}\right\}^{-1}$, close to (but never equal) to unity, ensuring that photons are in thermal equilibrium with the emitting substance and the emission by the surface is described by Planck's law (1) or (3).

In optically thin planar structures $\left(\ell_{\text {opt }}<\alpha>_{0}<\right.$ 1 ) with a flat emitting surface and refractive index $n_{\text {ref }}^{2} \gg 1$, a fraction $\sim\left(1-1 / 2 n_{\text {ref }}^{2}\right)$ ) of photons which are emitted internally outside the "escape cone" defined by Snell's law will be trapped by total internal reflection and eventually reabsorbed. In similar structures with light trapping, on the other hand, $a_{o} \cong$ $\ell_{\text {opt }}\langle\alpha\rangle_{\mathrm{o}}$ and the use of (11) then gives a reabsorption probability $r$ close to zero (Fig. 6). This, of course, is a direct result of Kirchhoff' s law which dictates that enhanced absorption implies enhanced emission, and has a consequence, for the operation of LEDs but also affects the voltage generated by solar cells that is discussed further below.

Another balance relation with application to photovoltaics was discovered by Rau ${ }^{30}$ and called the optoelectronic reciprocity (Fig. 4b ). The key result is that, under applied voltage $V$, the photon flux emitted by a solar cell /LED is equal to

$$
E Q E_{o} \Phi_{b b}\left(T_{o}\right) e^{q V / k_{B} T_{o}}
$$

where $E Q E_{o}$ is the external quantum efficiency of the solar cell under illumination with the black-body photon flux at temperature $T_{0}$. The (diode dark saturation) current produced under these conditions is obtained by dividing the photon flux (12) with the outcoupling factor or external quantum yield $Q_{e x t}$, equal to
$Q_{e x t}=(1-r) \frac{1}{1-r Q} Q$

where $Q$ is the internal quantum yield: the fraction of recombination events which result in internal photon emission, in contrast to $Q_{e x t}$ which refers to the external photon emission from the surface. The role of the three factors in (13) at various stages of photon emission is illustrated in Fig. 4b, showing also the link to charge-carrier reciprocity. ${ }^{31}$,

The various reciprocity laws help us understand the link between the incident and emitted photon flows and the open circuit voltage. Table 1 shows the additional terms which need to be added to the Shockley-Queisser voltage as a result of incomplete absorption, as described by Kirchhoff's law, due to a combined photon and carrier transport with photon recycling, and nonradiative recombination. The last column includes also the effect of carrier transport to junction. The result in this last column corresponds to the traditional treatment in solar cells theory, without allowance for photon reabsorption where all bulk recombination events result in carrier removal. This is appropriate in cells with low quantum yield, with crystalline silicon the principal application. The full result can be written as

$$
\begin{aligned}
q V_{o c}= & q V_{o c}^{S Q}+k_{B} T_{o} \ln \left(\frac{a_{S}}{a_{o}}\right) \\
& +k_{B} T_{o} \ln \left(Q_{e x t}\right)+k_{B} T_{o} \ln \left(\frac{I Q E_{S}}{I Q E_{o}}\right)
\end{aligned}
$$

where IQE's are the Internal Quantum Efficiencies defined in terms of the External Quantum Efficiencies and absorptivities by $I Q E=E Q E / a$, and the indices " $S$ " and " $O$ " refer to the incident solar and emitted lowtemperature light. The application of Eq. (14) will be illustrated in two particular cases: the effect of nonradiative recombination on voltage for an optically thick absorber, and the effect of the optical thickness of the absorber in the radiative limit $(Q=1)$. 
Figure 7 shows the reduction of the open circuit voltage as a function of the quantum yield $Q .{ }^{33}$ If the absorptivities for both the incident and emitted light are equal to unity, the reduction of voltage from $V_{o c}^{S Q}$ in the intermediate range of $Q$ comes from the third term on the right hand side of (14). In this range, the minority carrier density in the absorber is due to bulk and surface recombination; the former determined by the $Q$-term in the $Q_{e x t}(13)$. The total recombination rate increases linearly as a function of volume $\mathscr{V}$, giving rise to a term $-k_{B} T_{o} \ln \mathscr{V}$ in $V_{o c}$. Photon recycling is unimportant for these and smaller values of $Q$ as a single recombination event is sufficient to remove an electron - hole pair from the solar cell.

The voltage in this range of $Q$ can be written as $V_{o c}^{S Q}+k_{B} T_{o} \ln Q+k_{B} T_{o} \ln (1-r)$. The last term is sometimes interpreted ${ }^{34,35}$ as evidence that substantial photon recycling gives a large increase in the open circuit voltage, equal to the difference between the "classical" solar cell behaviour and the SQ limit. A more natural explanation using (11) with $a_{o} \cong 1$, however, is that photon recycling simply "mops up" the excess photons produced by recombination in the bulk in the high quantum yield limit, to comply with Planck's radiation law where emission occurs from the thin surface shell (Fig. 3) rather than from the full volume of the absorber.

For very low $Q$, the voltage is reduced by another contribution, which comes from the last term on the right hand side of (14). This region, together with the intermediate $Q$ values, is the subject of detailed discussion in the classical texts on solar cells in semiconductor physics $^{13,36,37}$. The operation of the cell is determined by solving the carrier transport equations in the absorber regions, with only electron-hole pairs generated by light within the diffusion length of the junction contributing to the power generated by the solar cell.
The variation of voltage with the luminescence quantum yield can be juxtaposed against this variation with the optical thickness in the radiative limit, as compared in Fig.8 for flat absorbers and for absorbers with perfect light trapping. The results - intended for illustration only - are shown in the "classical" limit, ignoring any features associated with light propagation in discrete waveguiding modes.

In the radiative limit, the voltage difference from the SQ limit is the Kirchhoff's law correction in the second column of Table 1. For such thin layers, the enhancement in absorption for incident sunlight is higher than for the emitted light producing, in general, an increase in the open circuit voltage. This does not mean, however, that the conversion efficiency of the solar cell is necessarily increased as the reduction in absorbed light must also be taken into account. In materials with a bandgap smaller that the maximum of the Shockley-Queisser curve $(\sim 1.3 \mathrm{eV})$, the absence of photon recycling increases the maximum efficiency by shifting the "effective" bandgap for photon emission towards higher values as is the case, for example, in silicon. ${ }^{38}$ In materials with higher bandgaps, the absence of photon recycling for emitted light reduces the maximum attainable efficiency.

In the thickness range $\ell_{\text {opt }}\langle\alpha\rangle_{0} \cong 1$, the absorptivity for the emitted light is some $2 n_{\text {ref }}^{2}$ times higher for absorbers with light trapping than for flat structures, equal to the ratio of the $(1-r)$ factors in the two cases. Taking $n_{r e f}=$ 3.54 - a typical value for inorganic semiconductors - we would therefore expect the difference between the two voltages to be around $k_{B} T_{o} \ln \left(2 n_{\text {ref }}^{2}\right) \cong 86 \mathrm{meV}$, higher for the flat absorber (with a higher reabsorption) than in the case of light trapping. The maximum difference is not far off the actual value $\sim 66$ $\mathrm{meV}$ which also includes a contribution from the absorptivity for the incident light. Interestingly, a similar result follows on thermodynamic grounds as isotropic scattering of a collimated beam increases the photon 
volume in the phase space by a factor of $2 n_{\text {ref }}^{2}$, leading to a corresponding increase in entropy and a reduction in voltage.

For extremely thin structures, the absorptivities of both the incident and emitted light are proportional to the mean absorption coefficient, and

$$
q V_{o c} \simeq q V_{o c}^{S Q}+k_{B} T_{o} \ln \left(\frac{\langle\alpha\rangle_{S}}{\langle\alpha\rangle_{o}}\right)
$$

This limit is, of course, unphysical for materials such as silicon but may be reached in molecular structures with layer thickness of one or few molecules.

\section{Thermodynamics of hot-carrier solar cells: learning from thermoelectricity}

We have so far used thermodynamics to help explain certain features of solar cell operation. But thermodynamics can also help propose new mechanisms, particularly in an area closest to the thermodynamicist's view point the hot carrier solar cells. Hot carrier solar cells were suggested first by Ross and Nozik in 1982 , aiming to eliminate the thermalization loss on photon cooling. ${ }^{39}$ Their model, which - in common with the conventional solar cells - conserves the number of photons, is summarized in Fig. 9a.

Electron-hole pairs are generated by light in an absorber and come to a mutual thermal equilibrium at a temperature $T_{H}>T_{o}$ by virtue of a limited or no interaction with lattice vibrations. Charge separation occurs whilst the charge carriers are cooled to the ambient temperature, again avoiding heat loss to extraneous degrees of freedom. In the Ross and Nozik model this cooling - which has to occur at constant entropy of the charge carriers - takes place via "selective energy contacts": only electrons and holes in a very narrow energy range are extracted to the external contacts.
In a more general approach, the thermodynamics of the Ross and Nozik model is shown schematically in Fig. 9b, avoiding some of the issues that have been highlighted in ref. ${ }^{40} \mathrm{In}$ the absence external interactions the absorber temperature and chemical potential are determined by the photon balance between absorption and emission, taking into account extraction for energy conversion. It therefore follows that the emitted photons - which share the charge carrier - characteristics - are described by the elevated temperature of the absorber.

Charge carriers produce current by being extracted into an ideal Carnot - like converter where electron - hole pairs are separated and cooled to room temperature. The second law of thermodynamics demands that, whilst the cooling can proceed adiabatically without energy exchange with the surroundings, heat has to be rejected into a reservoir at temperature $T_{o}$ to balance the entropy gain in the absorber.

Most experimental effort to-date has been devoted to finding suitable materials and structures for practical implementation. Although an ideal hot-carrier solar cell can operate with a zero energy gap, the search for suitable materials has been almost invariably focused on semiconductors where attempts to slow down thermalization include, for example, the use of quantum wells ${ }^{41,42,43,44,45}$ with electron-phonon interaction reduced by the formation of a "phonon bottleneck". Analyses of energy selective contacts includes, for example, quantum dots-based tunnelling structures. ${ }^{43,46,47}$ A practical demonstration, however, is still lacking and alternative approaches have been proposed. ${ }^{44,48,49} 50$

Thermodynamics can help to tackle each of the two challenges highlighted in the Ross and Nozik work. Since the best conventional solar cells already capture all, or almost all, the available solar flux for a given bandgap, the principal aim of hot carrier solar cells is to increase the open circuit voltage. We shall show below how thermodynamics can cast 
new light on how a hot carrier converter can achieve this goal based on the principles of thermoelectric conversion. The challenge to find new materials with long excited states and reduced energy dissipation by thermalization has been addressed recently in ${ }^{51}$ and will be discussed further elsewhere.

To pave the way for describing how a thermoelectric hot carrier solar cell would work, it is useful to review briefly the fundamentals of semiconductor thermoelectrics. A standard thermoelectric circuit to generate current, formed by segments of a p and n-type semiconductor separated by p-n junctions, is shown in Fig. 10a. One junction is heated to a temperature $T_{h}$ whilst the other remains at the ambient temperature $T_{o}$, driving current $I$ in the circuit. A thermoelectric voltage generator is based on the principles of the Seebeck effect (Fig. 10b). Standard theory gives the thermoelectric voltage, or thermopower, in the form

$$
V=\left(T_{h}-T_{o}\right)\left(\diamond_{n}-\vartheta_{p}\right)
$$

where $\iota_{n}$ and $\iota_{p}$ are the Seebeck coefficients of the $\mathrm{n}$ and $\mathrm{p}$ type materials, assumed here to be constant.

In thermodynamic terms, energy / voltage is produced by the absorption of heat $Q_{j}\left(T_{h}\right)$ at the high temperature junction from a reservoir at temperature $T_{h}$ and the voltage detector acts as an implied junction at temperature $T_{o}$, rejecting heat $Q_{j}\left(T_{o}\right)$. The circuit in Fig.10b therefore works as a Carnot engine, the energy $q V$ representing work done in one cycle corresponding to the passage of an electron through the circuit in Fig. 10b. In terms of chargecarrier thermodynamics discussed in Sec. 1, heat $Q_{j}\left(T_{h}\right)$ is equal to the energy needed to excite an electron from the valence band on the p-side to the conduction band on the nside:

$$
Q_{j}\left(T_{h}\right)=E_{g}+2 u_{t_{h}}-q V_{b i}\left(T_{h}\right)
$$

(Fig. 11; see also ref. 52) where we noted that the thermal energy $u_{t}$ is equal for electrons and holes. Heat / thermal energy equal to

$Q_{s n}\left(T_{o}\right)+Q_{c p}\left(T_{o}\right)=Q_{j}\left(T_{o}\right)$

is rejected at the contacts to the voltage detector which replace the low-temperature p$n$ junction. In an ideal thermoelectric converter, maximum work (or voltage) is produced with the Carnot efficiency:

$q V=Q_{j}\left(T_{h}\right)-Q_{j}\left(T_{o}\right)=\left(1-\frac{T_{o}}{T_{h}}\right) Q_{j}\left(T_{h}\right)$

Substituting from (17) and (18), using the standard result for the built in voltage and neglecting the variation of the effective densities of states with temperature we can verify that Eqs. (16) and (19) give identical results for the voltage. As shown in standard texts, ${ }^{53}$ we have used here the result that the Seebeck coefficients are equal to the carrier entropies, with an appropriate sign (negative for n-type, positive for p-type), divided by the elementary charge.

This interpretation provides a way forward to hot carrier photovoltaics. All that needs to be done is to replace the high-temperature heat by illumination from the Sun: a heat source at temperature $T_{S}$, in other words, exciting the solar cell directly by absorbing the solar heat / radiation at the high-temperature junction. Because of the strong electric field, the electron transit time across the junction is very short (of the order of picoseconds), making it possible for charge separation to occur before thermalization. A good hot-carrier thermoelectric solar cell would then complement the high temperature absorption / charge separation step with a subsequent controlled cooling in the two "adiabatic" $\mathrm{n}$ and p segments. Learning from colleagues working in thermoelectricity, the aim would be to conserve carrier entropies (or Seebeck coefficiens $\diamond_{n}$ and $\diamond_{p}$ ). In a practical device, the challenge will additionally be to limit losses 
by heat conduction (and, away from open circuit, also by Joule heating).

We may compare such thermoelectric converter with a traditional solar cell (Fig. 12). Tracing the various steps of the conversion process, light absorption creates a hot electronhole gas (Fig. 12a) where electrons and holes are in mutual equilibrium at a temperature $T_{h}$. The photogenerated pairs rapidly thermalize / cool, accompanied by heat loss and entropy production. We have seen in Sec. 1 that subsequent charge separation at the junction is an isothermal process with little or no energy loss where electrons and holes acquire different chemical potentials, and the difference gives the open-circuit voltage (Fig. 12b). Just as in a thermoelectric, the proposed model for hot carrier solar cell therefore foresees a voltage loss of $V_{b i}\left(T_{h}\right)$ due to the charge separation step.

The combination of photovoltaic and thermoelectric conversion therefore offers the key advantages of combining a good voltage generator (a thermoelectric) with a good current generator (a solar cell). Thermoelectrics manifest relatively low power conversion efficiencies but the voltage efficiency (defined in thermodynamic terms outlined above) is high: the thermopower, or energy output, of many semiconductor thermoelectric devices is close to the bandgap, even under modest temperature differences. A high voltage produced by thermoelectrics indicates a good synergy with photovoltaics where the current produced by a good solar cell can approach $100 \%$ of the theoretical maximum.

A key advantage of the proposed scheme which relies of generating hot charge carriers directly in the field-containing region of the junction over the Ross and Nozik mechanism is the rapid charge separation, obviating the need to search for materials with slow thermalization rates. This feature offers a characteristic fingerprint to recognise such solar cell: the optical emission. Since the rate of radiative recombination rate increases strongly with increasing temperature, most photons in such a hot carrier cell would be emitted with a temperature determined by the incident light, in keeping with the more general view outlined in Sec. 1. This is in sharp contrast with homogeneous semiconductors or conventional solar cells where emission occurs after thermalization, at the temperature of the material.

\section{Conclusion}

In this brief overview, we have outlined a number of areas where thermodynamics is not only an integral part of the theoretical framework behind photovoltaics but can broaden the scope to pave the way towards new ideas and mechanisms. By applying classical thermodynamics to light we obtained a detailed picture of fundamental losses in PV conversion, in particular, losses incurred in thermalization and optical entropy generation under one sun illumination. Energy flows which make up the conversion process in a solar cell are subject to a number of detailed balance and reciprocity laws which, in turn, determine the voltage generated by the cell. Through a closer analysis of these laws we have been able to provide a picture of the delicate interplay between absorption and emission of light, voltage and the role played by processes such as photon recycling and light trapping / management. Heat flows and thermodynamics are also an integral part of charge carrier transport in semiconductors, in particular, in p-n junctions. This is particularly true in phenomena such as thermoelectricity. We have shown how this type of analysis can be used to advantage to reduce the thermalization losses and the opportunities it provides to take a fresh look at the workings of hot carrier solar cells. It is interesting to note that thermoelectric conversion was suggested as a model (albeit for conventional solar cells) already by Müser in $1957 .{ }^{54}$ 


\section{Acknowledgement}

Centre for Advanced Photovoltaics is supported by the Czech Ministry of Education, Youth and Sport.

CZ.02.1.01/0.0/0.0/15_003/0000464.

\section{Appendix A: Radiative balance between photon emission from surface and in volume}

We outline here a simple derivation of Eq. (10) which underpins the arguments of Sec. 2. Assuming, for simplicity, that the emitting medium is surrounded by vacuum, the monochromatic photon flux emitted per unit time by a surface is given by

$\Phi_{\text {surf }}=e \Phi_{b b}=e \varnothing \phi_{o}$

where $e$ is Kirchhoff's emissivity, $\Phi_{b b}$ is the black-body emission rate, $\phi_{o}$ is the black-body photon flux per unit area per unit solid angle in vacuum, and $\mathscr{E}$ is the étendue of the emitted beam (see, for example, ref. 11). The rate of internal photon emission is

$\Phi_{\text {vol }}=\varepsilon \mathscr{Y}=4 \pi n_{\text {ref }}^{2}\langle\alpha\rangle_{o} \phi_{o} \gamma$

where $\varepsilon$ is the photon (rather than energy) analogue of Planck's emission coefficient (rate of photon emission per unit volume).
Equation (A2) follows from the detailed balance between absorption and emission in the volume as formulated by Planck ${ }^{24}$. For semiconductors, Eq. (A2) was obtained by van Roosbroeck and Shockley ${ }^{27}$ in the form

$$
B n_{i}^{2}=4 \pi n_{r e f}^{2}\langle\alpha\rangle_{o} \phi_{o}
$$

where $B$ is the rate constant for radiative recombination and $n_{i}$ is the intrinsic carrier concentration.

Since all internally emitted photons will leave the medium unless removed by reabsorption (with average probability $r$ ), we can write

$$
\Phi_{\text {surf }}=(1-r) \Phi_{\text {vol }}
$$

Substituting from (A1) and (A2), using Kirchhoff's law to replace emissivity $e$ by absorptivity $a_{o}$, and defining a length $\ell_{\text {opt }}=4 \pi n_{\text {ref }}^{2} \% / \&$ now gives Eq. (11) in the text where we noted that, for a planar structure of thickness $d, \quad \ell_{\text {opt }}$ reduces to $4 n_{\text {ref }}^{2} d$. 


\section{References}

1. V.F. Chel'tzov, Interaction of two-band semiconductor with radiation field, Solid State Commun. 9, 1343 (1971)

2 H.B. Bebb and E.W. Williams, Photoluminescence I: Theory. In Willardson RK., Beer AC. eds. Semiconductors and Semimetals, Vol. 8, Elsevier, 1972.

3 P. Würfel, The chemical potential of radiation J. Phys. C: Solid St. Phys. 15, 3967 (1982)

4 W. Shockley and H.J. Queisser, Detailed balance limit of efficiency of p-n junction solar cells, J. Appl. Phys. 32, 510 (1961)

5 C. Kittel and H. Kroemer, Thermal Physics $\left(2^{\text {nd }}\right.$ ed), W.H. Freeman, New York (1980).

$6 \quad$ A. Rose, Photovoltaic effect derived from the Carnot cycle, J. Appl. Phys. 31, 1640 (1960).

7 T. Markvart, Solar cell as a heat engine: energy-entropy analysis of photovoltaic conversion, physica stat. sol. (a), 205, 2752 (2008)

8 G. Gouy, Sur l'énergie utilizable, Journal de Physique, 8:501 (1889)

9 A. Stodola, Die Kreisprozesse der Dampfmaschine, Zeitschr. d. Vereines deutscher Ingeniuere, 32, 1086 (1898).

10 T. Markvart, Thermodynamics of losses in photovoltaic conversion, Appl. Phys. Lett. 91, 064102, (2007).

11 T. Markvart, The thermodynamics of optical étendue J. Opt. A: Pure Appl. Opt. 10, 015008 (2008)

12 W. Shockley, Electrons and Holes in Semoconductors, Van Nostrand Inc. (1951)

13 S.M. Sze and Kwok K. Ng, Physics of Semiconductor Devices ( $3^{\text {rd }}$ ed.), John Wiley \& Sons, Hoboken (2007).

14 R.A. Smith, Semiconductors, Cambridge U.P., London, 1979 ( $2^{\text {nd }}$ ed)

15 K. Seeger, Semiconductor Physics, Springer, Berlin, 1999 ( $7^{\text {th }}$ ed)

16 P. Würfel, Physics of Solar Cells, Wiley, Weinheim (2005)

17 U. Würfel, A. Cuevas and P. Würfel, Charge carrier separation in solar cells, IEEE J. Photovolt. 5, 461 (2015)
18 J. Brendel and R. Peibst, Contact selectivity and efficiency in crystalline silicon photovoltaics, IEEE J. Photovolt. 6, 1413 (2016)

19 S.R. de Groot and P. Mazur, Nonequilibrium Thermodynamics, NorthHolland, Amsterdam (1962)

20 H.J. Goldsmid, Applications of thermoelectricity, Methuen \& Co, London, 1960.

21 C. Kittel, Introduction to Solid State Physics ( $8^{\text {th }}$ ed.), Wiley, Hoboken (2005),

${ }^{22}$ M.R. Peterson and B.S. Shastry, Kelvin formula for thermopower, Phys. Rev. B 82, 195105 (2010).

23 T. Markvart, From steam engine to solar cells: can thermodynamics guide the development of future generations of photovoltaics? Wiley Interdisciplinary Reviews: Energy and Environment 5, 543 (2016)

24 M. Planck, The Theory of Heat Radiation. Dover, New York, 1991.

25 E. H. Kennard, On the thermodynamics of fluorescence, Phys. Rev. 11, 29 (1918); On the interaction of radiation with matter and on fluorescent exciting power, Phys. Rev. 28, 672 (1926).

26 B.I. Stepanov, A universal relation between the absorption and luminescence spectra of complex molecules, Sov. Phys.Dokl. 112, 81 (1957); Correlation between the luminescence and absorption spectra of complex molecules, Izv. Akad. Nauk SSSR 22, 1357 (1958).

27 W. van Roosbroeck and W. Shockley, Photon-radiative recombination of electrons and holes in germanium, Phys. Rev. 94, 1558 (1954)

${ }^{28}$ W. Dumke, Spontaneous radiative recombination in semiconductors, Phys. Rev. 105, 139 (1957)

29 R.K. Ahrenkiel et al, Ultralong minoritycarrier lifetime in epitaxial GaAs by photon recycling, Appl. Phys. Lett. 55, 1088 (1989).

30 U. Rau, Reciprocity relation between photovoltaic quantum efficiency and electroluminescent emission of solar cells, Phys. Rev. B 76, 085303 (2007). 
31 C. Donolato, A reciprocity theorem for charge collection, Appl. Phys. Lett. 46, 270 (1985)

32 T. Markvart, Relationship between dark carrier distribution and photogenerated carrier collection in solar cells. IEEE Trans. Electron. Dev. 43, 1034 (1996)

33 T. Markvart, Open circuit voltage and reciprocity in solar cells, IEEE J. Photovolt. 8, 67 (2017).

34 A. Martí, J.L. Balenzategui \& R.F. Reyna, Photon recycling and Shockley's diode equation, J. Appl. Phys. 82, 4067 (1997).

35 T. Kirchartz, F. Staub and U. Rau, Impact of photon recycling on the open-circuit voltage of metal halide perovskite solar cells, ACS Energy Letters, 1, 731 (2016).

${ }^{36}$ M. A. Green, Solar Cells - Operating Principles, Technology and System Application. Kensington, Australia: University of NSW, 1992.

37 A.L Fahrenbuch and R.H. Bube, Fundamentals of Solar Cells. Photovoltaic Solar Energy Conversion. Academic Press, London (1983)

38 T. Markvart et al, Harvesting sunshine: solar cells, photosynthesis and the thermodynamics of light, $27^{\text {th }}$ European PVSEC, Frankfurt, 2012.

39 R.T. Ross and A.J. Nozik, Efficiency of hot-carrier solar energy converters, J. Appl. Phys. 53, 3813 (1982).

40 P. Würfel, A.S. Brown, T.E. Humphrey and M.A. Green, Particle conservation in the hot-carrier solar cell, Prog. Photovolt. Res. Appl. 13, 277 (2005)

${ }^{41}$ Y. Rosenwaks et al., Hot-carrier cooling in GaAs: Quantum wells versus bulk. Physical Review B,. 48, 14675 (1993)

42 A. Nozik et al., Dependence of hot carrier luminescence on barrier thickness in GaAs/ AlGaAs superlattices and multiple quantum wells. Solid St. Comm, 75, 297 (1990)

43 G. Conibeer et al., Progress on hot carrier cells. Solar Energy Materials and Solar Cells, 93, 713-719 (2009)

44 J. Rodière et al., Experimental evidence of hot carriers solar cell operation in multiquantum wells heterostructures. Applied Physics Letters, 106, 183901 (2015).
H. Esmaielpour, V. R. Whiteside, J.

Tang et al, Suppression of phononmediated hot carrier relaxation in type-II InAs/AlAs $\mathrm{Sb}_{1-\mathrm{x}}$ quantum wells: a practical route to hot carrier solar cells, Prog. Photovolt: Res. Appl. 24, 59 (2016)

46 A. Le Bris and J.-F. Guillemoles, Hot carrier solar cells: Achievable efficiency accounting for heat losses in the absorber and through contacts. Applied Physics Letters, 97, 113506 (2010.).

47 D. König, et al., Hot carrier solar cells: Principles, materials and design. Physica E: Low-dimensional Systems and Nanostructures, 42, 2862 (2010)

48 D.J. Farrell, et al., A hot-carrier solar cell with optical energy selective contacts. Applied Physics Letters, 99, 111102 (2011)

49 I. Konovalov and V. Emelianov, Hot carrier solar cell as thermoelectric device. Energy Sci. \& Eng., 5, 113 (2017).

50 D.T Nguyen, L. Lombez, F. Gibelli, S. Boyer-Richard, A. Le Corre, O. Durand \& J.F. Guillemoles, Quantitative experimental assessment of hot carrier-enhanced solar cells at room temperature. Nature Energy, 3(3), 236 (2018).

51 B. Dzurnak et al, Hot photons and opencircuit voltage in molecular absorbers, submitted to Semicon. Sci. Technol.

52 J. Tauc, The share of thermal energy taken from the surroundings in the electroluminescent energy radiated from a p-n junction, Czech. J. Phys. 7, 275 (1957).

53 H.B. Callen, Thermodynamics and an Introduction to Thermostatistics, Wiley, New York (1985).

54 H.A. Müser, Thermodynamische Behandlung von Elektronenprozessen in Halbleiter-Randschichten, Z. Physik. 148, 380 (1957). 


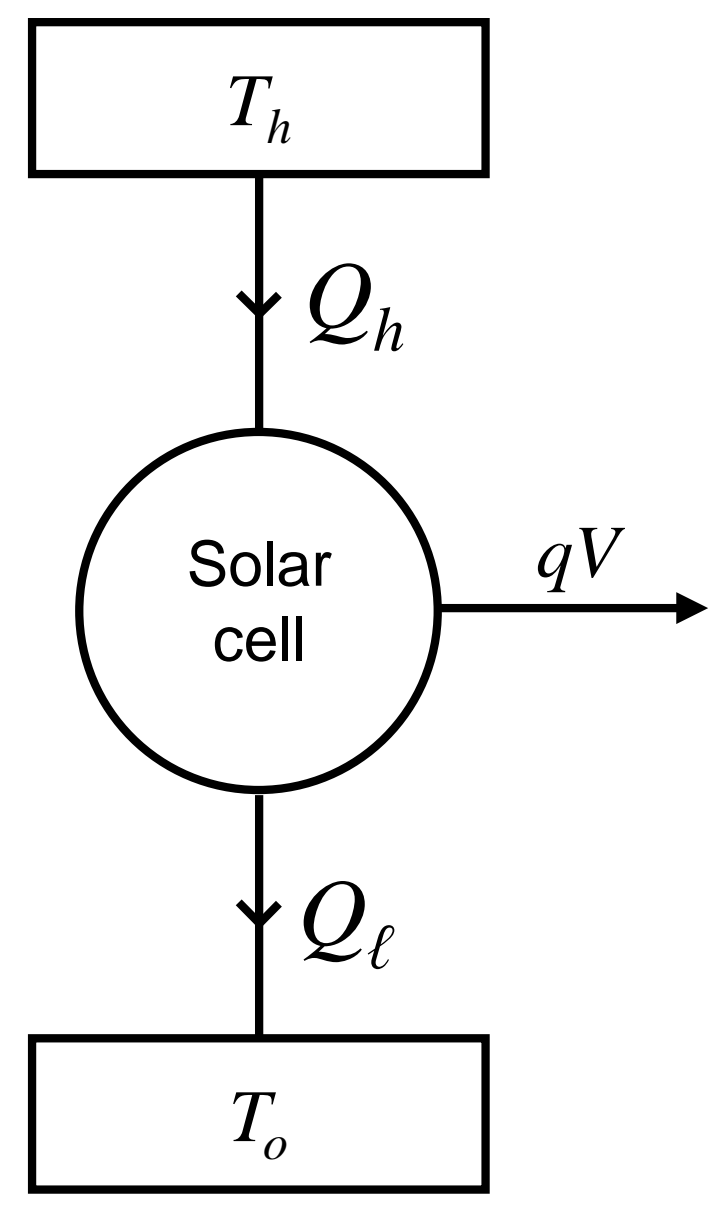

Fig. 1. Solar cell as a heat engine. 


\begin{tabular}{|r|c|c|c|c|}
\hline & Shockley \& Queisser & Kirchhoff & \multicolumn{2}{|c|}{ Optoelectronic / charge-carrier reciprocity } \\
\hline Incident & $\Phi_{b b}\left(T_{S}\right)=\mathscr{E}_{S} \phi_{b b}\left(T_{S}\right)$ & $a_{S} \Phi_{b b}\left(T_{S}\right)$ & $a_{S} \Phi_{b b}\left(T_{S}\right)$ & $E Q E_{S} \Phi_{b b}\left(T_{S}\right)$ \\
\hline Emitted & $\Phi_{b b}\left(T_{o}\right)=\mathscr{E}_{o} \phi_{b b}\left(T_{o}\right)$ & $a_{o} \Phi_{b b}\left(T_{o}\right)$ & $a_{o} \Phi_{b b}\left(T_{o}\right)$ & $E Q E_{o} \Phi_{b b}\left(T_{o}\right)$ \\
\hline Comment & $q V_{o c}^{S Q}$ & $+k_{B} T_{o} \ln \left(\frac{a_{S}}{a_{o}}\right)$ & $+k_{B} T_{o} \ln \left(Q_{e x t}\right)$ & $+k_{B} T_{o} \ln \left(\frac{I Q E_{S}}{I Q E_{o}}\right)$ \\
\hline & Radiative limit & $\begin{array}{c}\text { With photon } \\
\text { recycling / } \\
\text { nonradiative } \\
\text { recombination }\end{array}$ & $\begin{array}{c}\text { With carrier } \\
\text { transport to } \\
\text { junction }\end{array}$ \\
\hline
\end{tabular}

Table 1. The relationship between the various reciprocity laws for photon emission and absorption, and the open circuit voltage generated by a solar cell. $\mathscr{E}$ and $I Q E=E Q E / a$ are the étendues and internal quantum efficiencies, and the subscripts " $S$ " and " $O$ " of all relevant quantities correspond to the incident solar and emitted low-temperature light. 


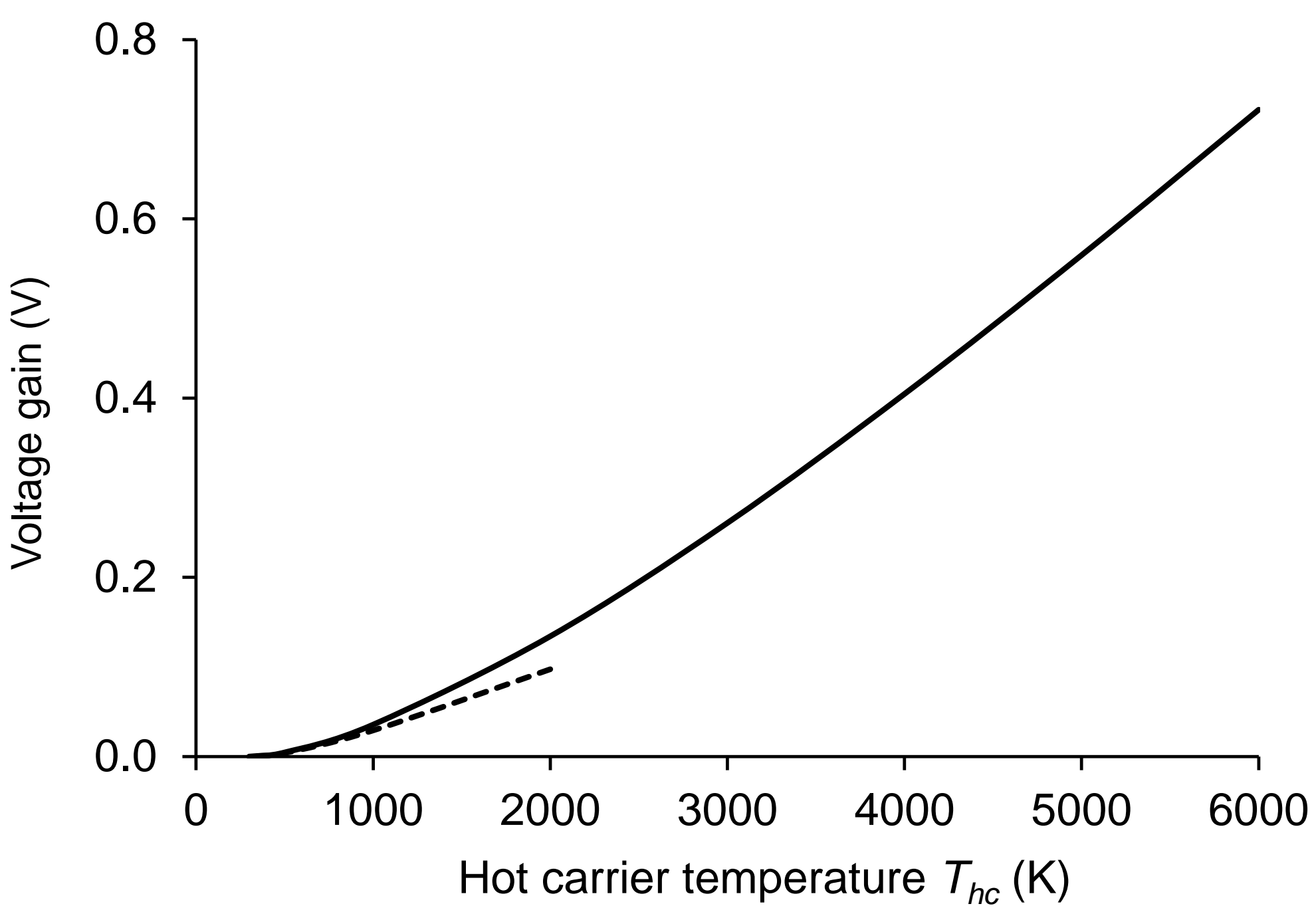

Fig. 2. The voltage gain in a hot carrier solar cell with a bandgap of $E_{g}=1.12 \mathrm{eV}$ corresponding to silicon where photons / carriers thermalize irreversibly from temperature $T_{S}$ to $T_{h c}$ (full line), followed by a reversible voltage generation / energy extraction when cooled to $T_{0}$. Also shown is an approximation for an ideal photon gas useful for smaller temperature differences $T_{h c}-T_{o}$ (dashed line). ${ }^{7}$ 


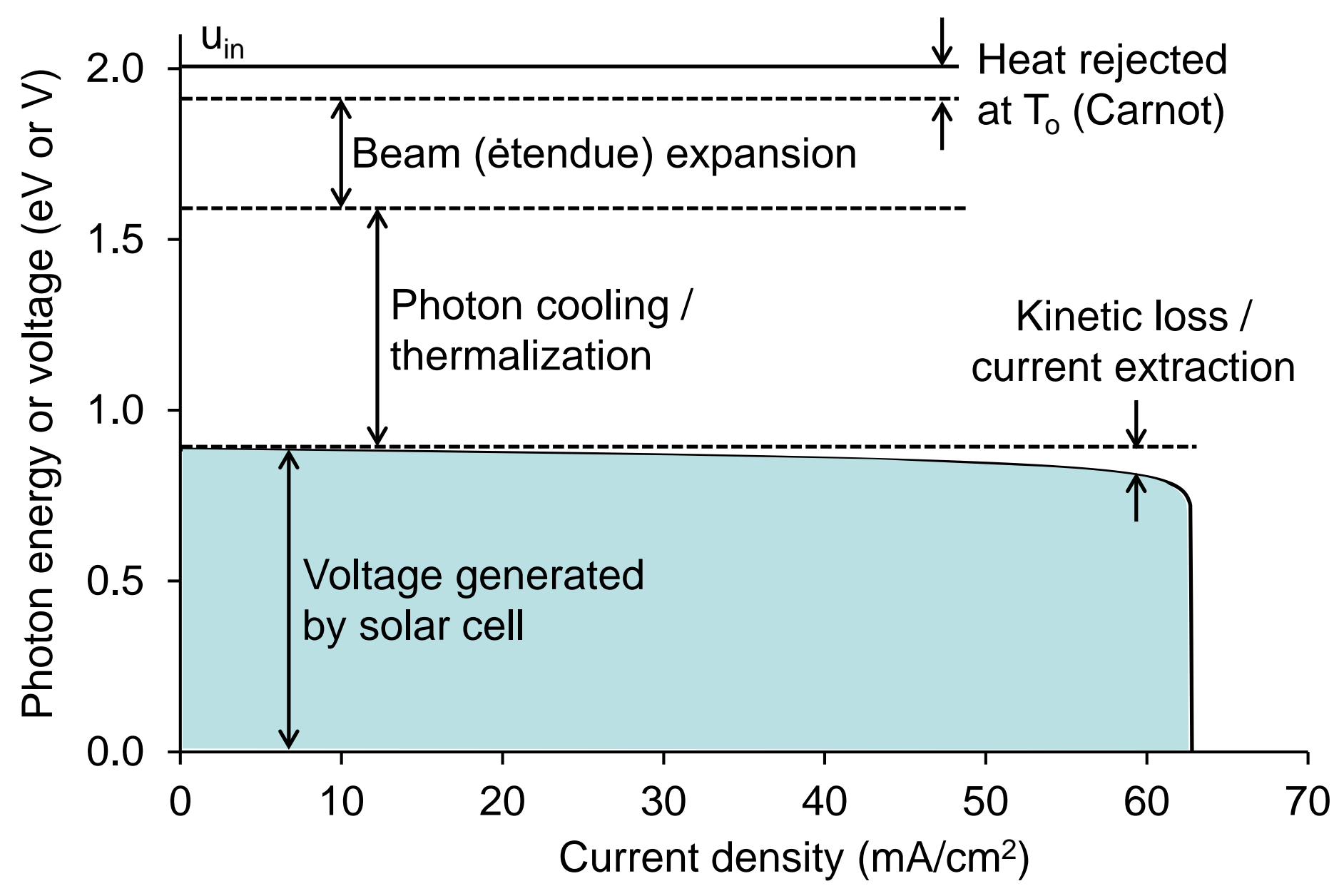

Fig. 3. The "thermodynamic" (V-I) characteristic of a crystalline silicon solar cell illustrating the fundamental losses in PV conversion. 


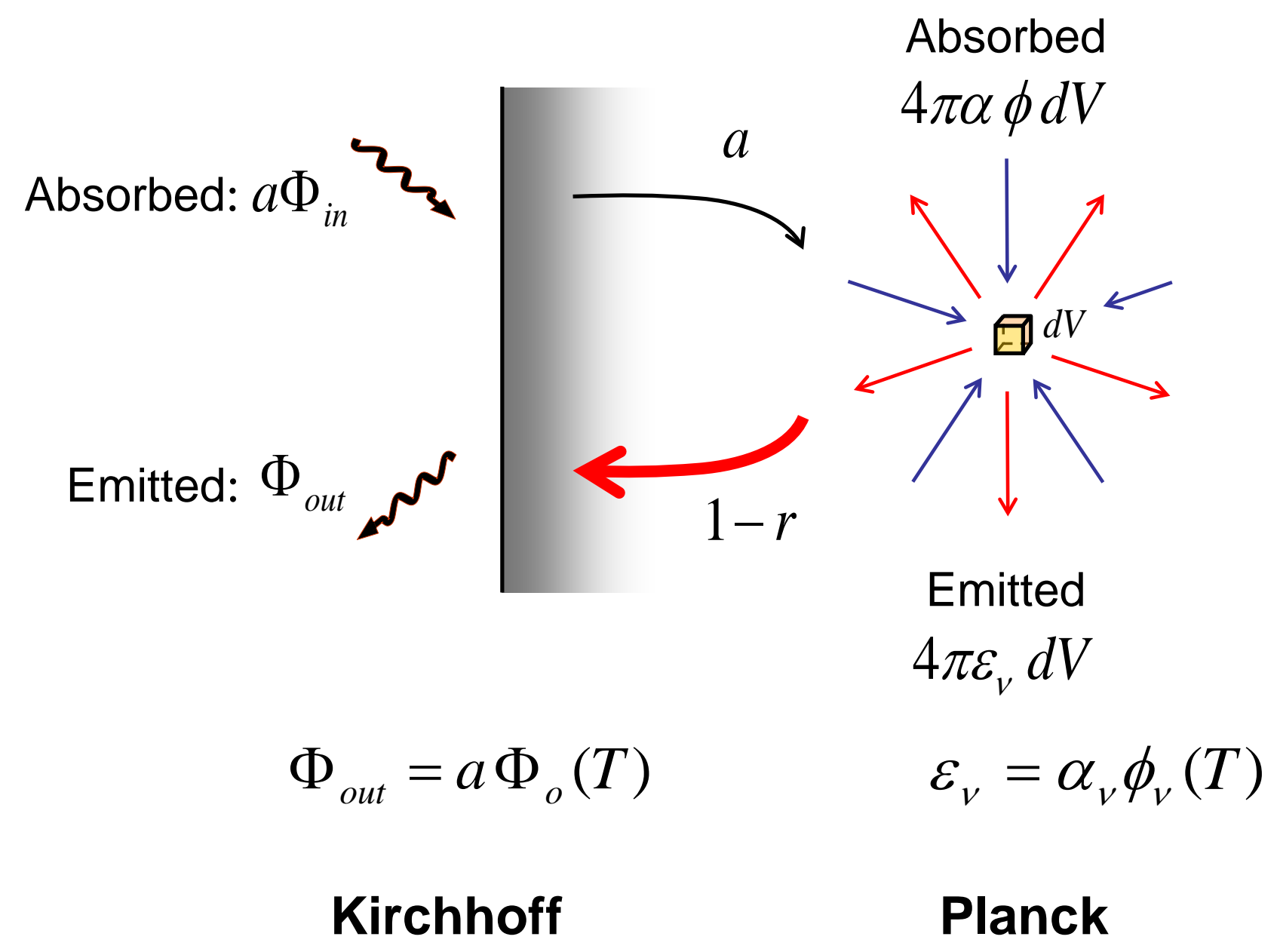

Fig. 4. The balance between absorption and emission of light. (a) The laws of Kirchhoff and Planck which apply to thermal radiation emitted by a surface and in volume, showing also photon recycling which adds an additional balance between the two. 


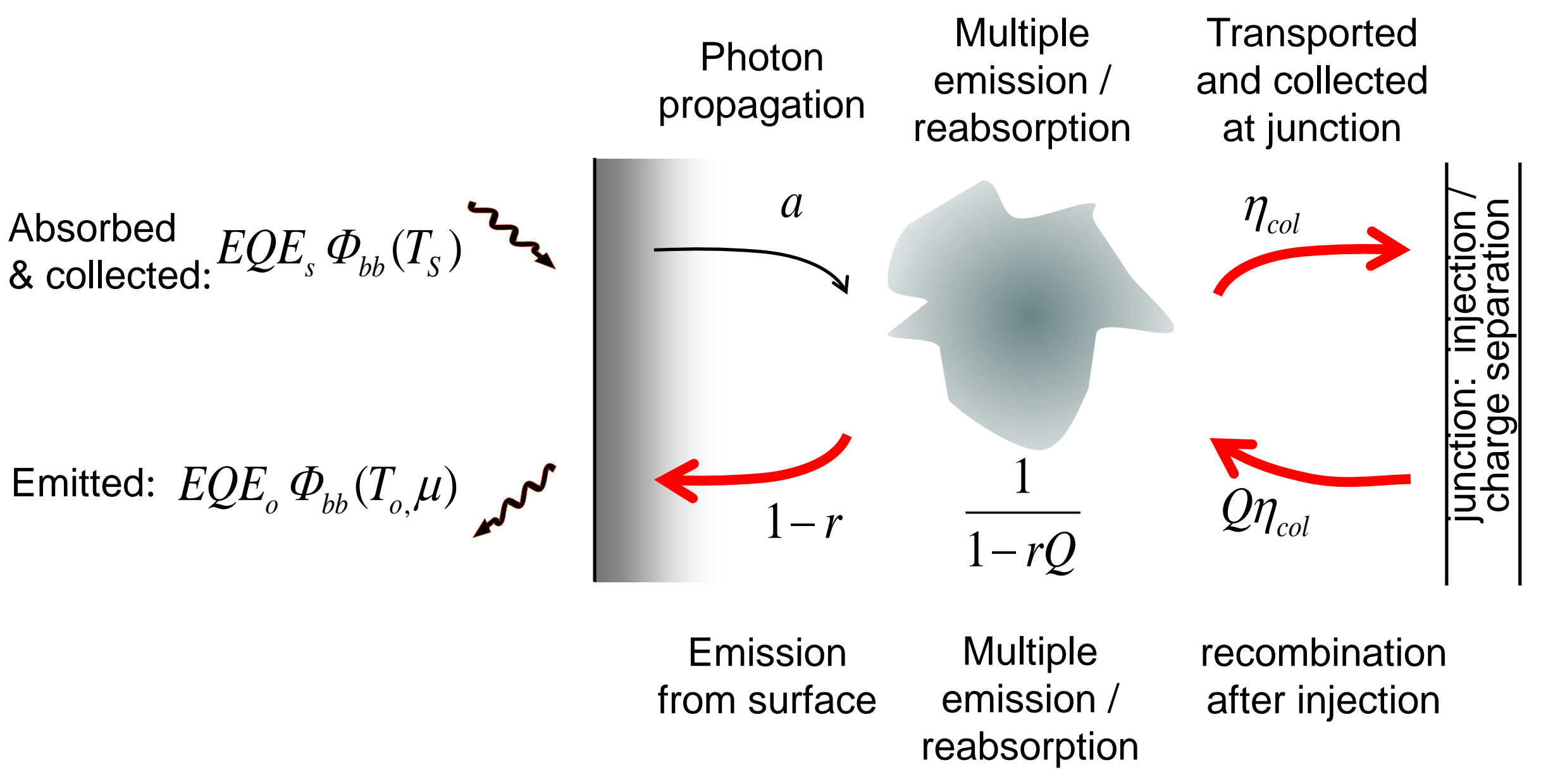

\section{Optoelectronic / carrier transport reciprocity}

Fig. 4(b) The optoelectronic and charge-carrier reciprocity relations which govern the absorption and emission of light in solar cells or LEDs. 


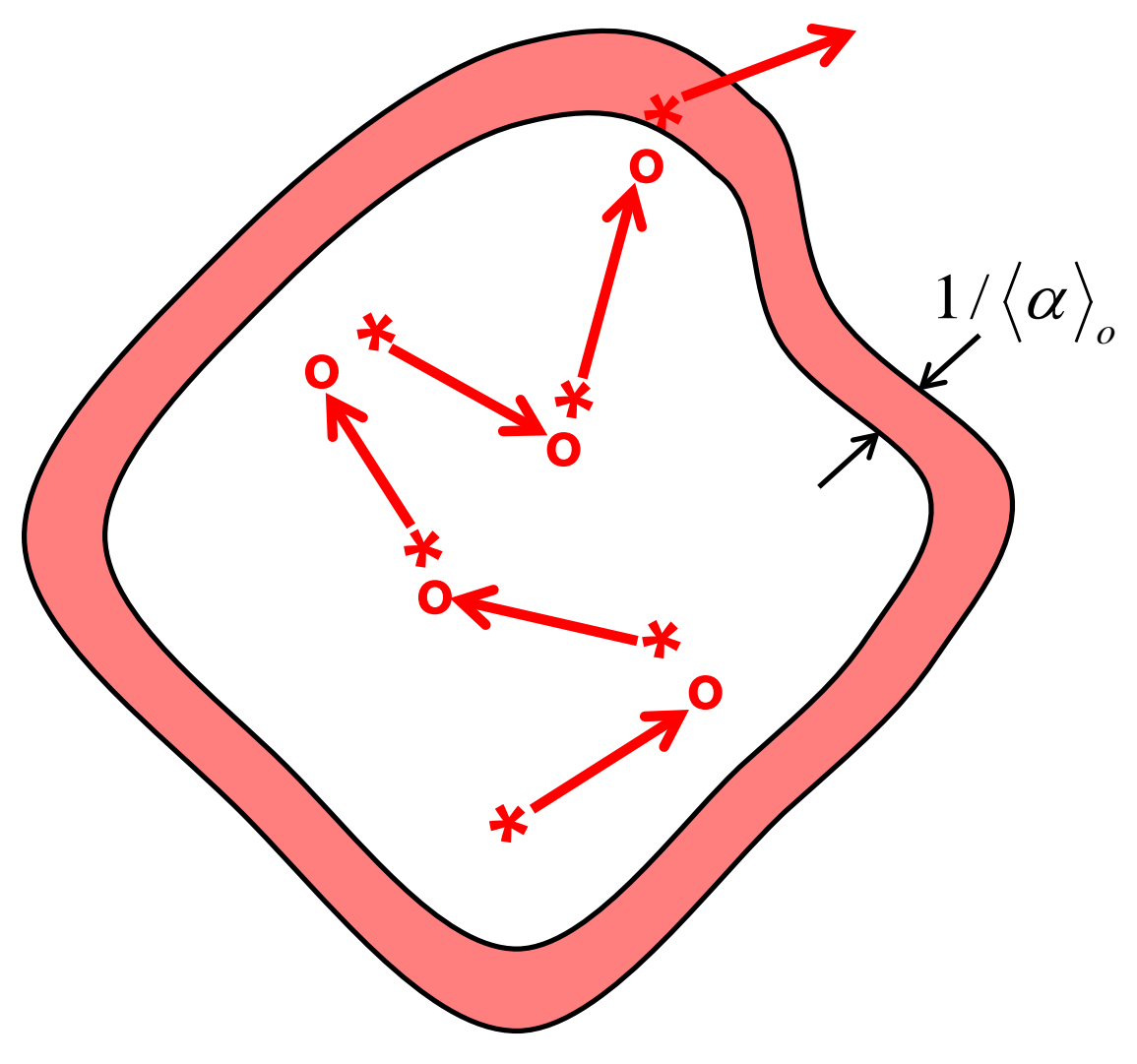

Fig. 5. Photon emission (stars) and re-absorption (circles) in optically thick emitters. Only photons emitted in a thin shell of thickness of the order of the inverse absorption coefficient at temperature $T_{o}$ near the surface ("the photosphere", to use an analogy with the Sun) will be emitted to the exterior. 


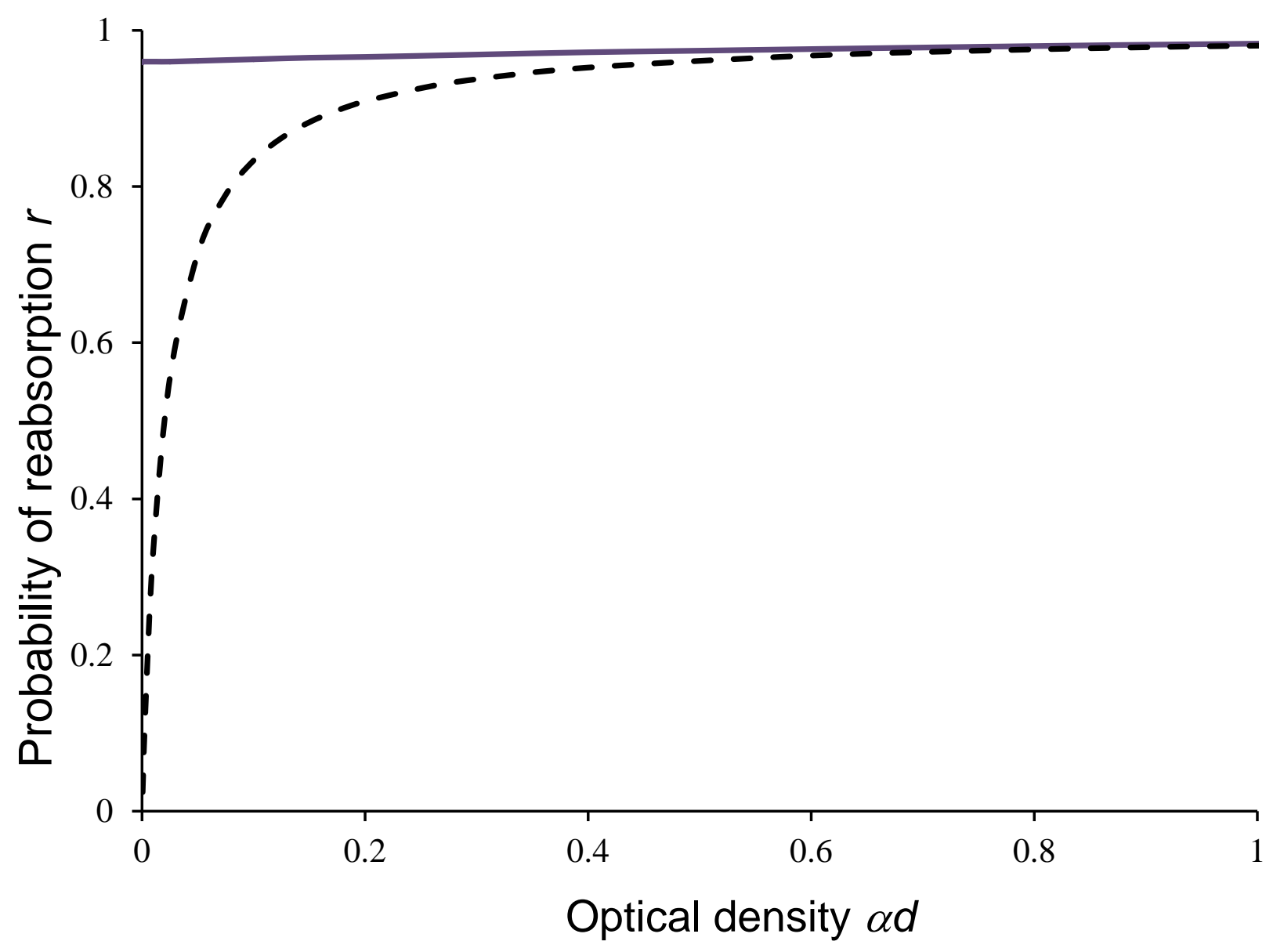

Fig. 6. The probability of reabsorption in flat absorbers of thickness $d$ (full line), and absorbers with perfect light trapping (dashed line). The graphs are for refractive index 3.54, typical for inorganic semiconductors. 


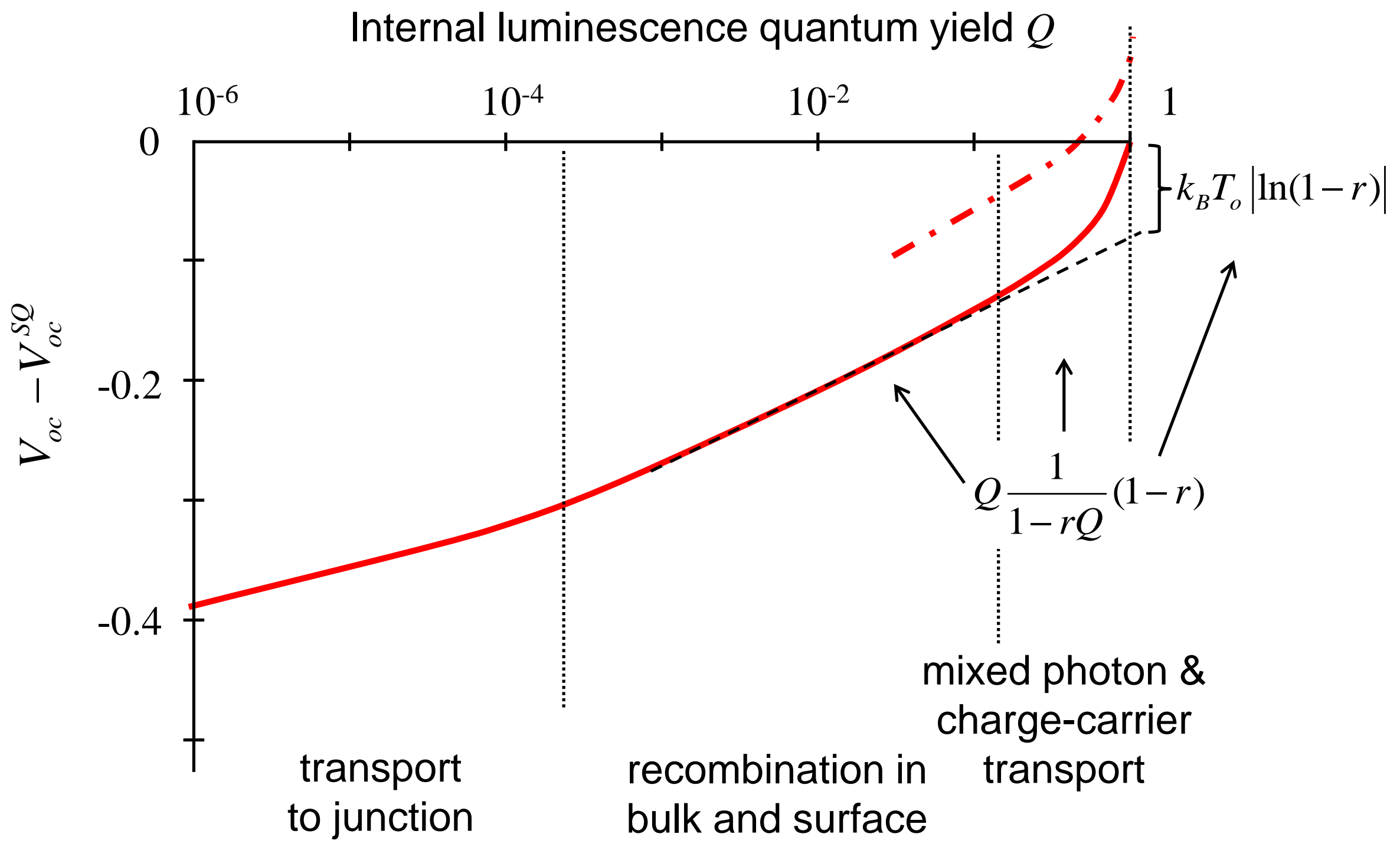

Fig. 7. The reduction of the open circuit voltage from the Shockley-Queisser limit for an optically thick absorber as a function of the quantum yield $Q$ (full line). For comparison, the dash-dot line shows the effect on voltage of reducing the optical thickness below the absorption depth $1 /<a>{ }_{0}$. 


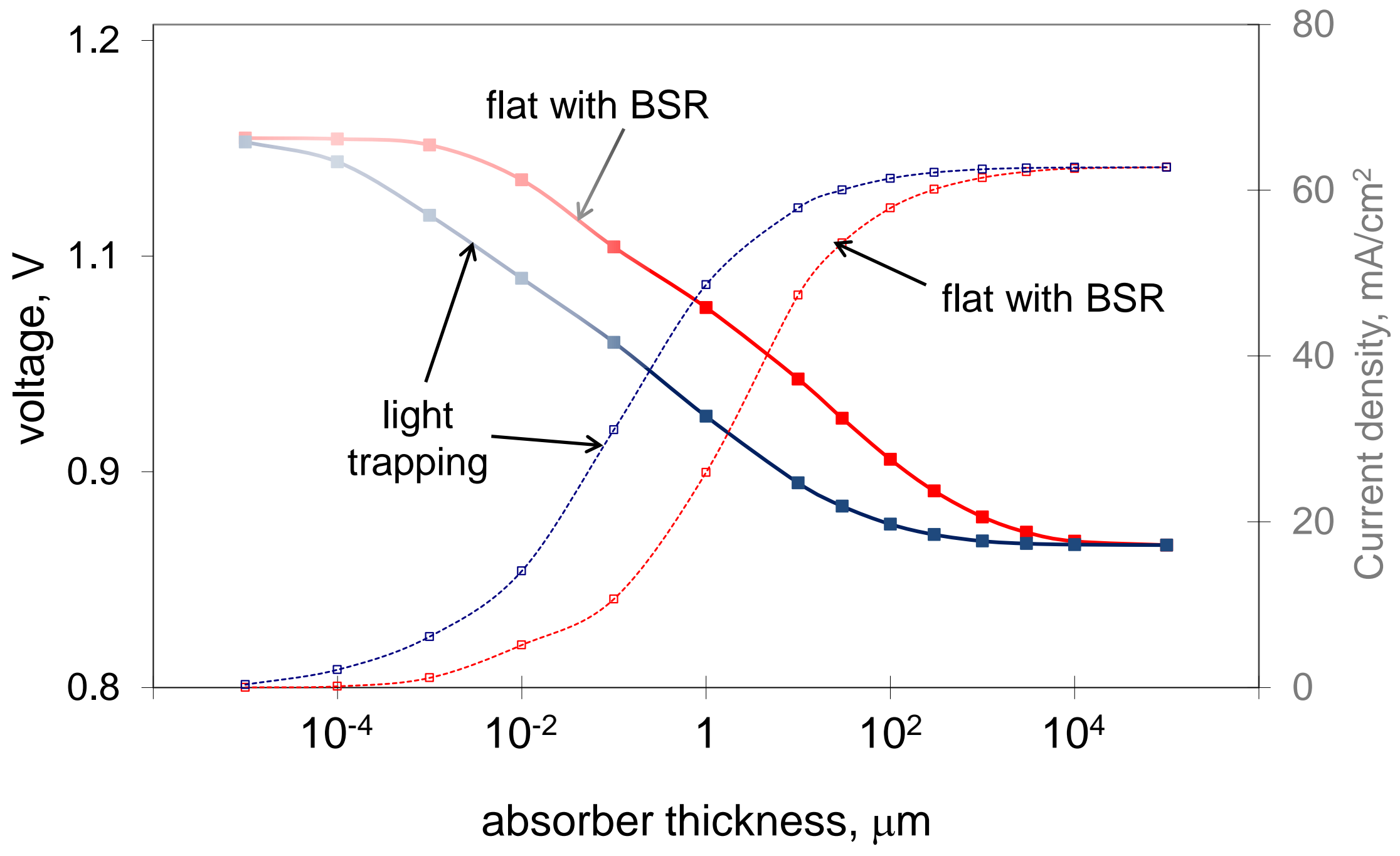

Fig. 8. The voltage in thin silicon absorbers, given by the full lines, for smooth flat absorbers and for absorbers with perfect light trapping. These lines are extrapolated to a hypothetical limit of ultrathin layers where voltage would be expected to follow Eq. (15). For comparison, the photogenerated current density is also shown (faint dashed lines). 


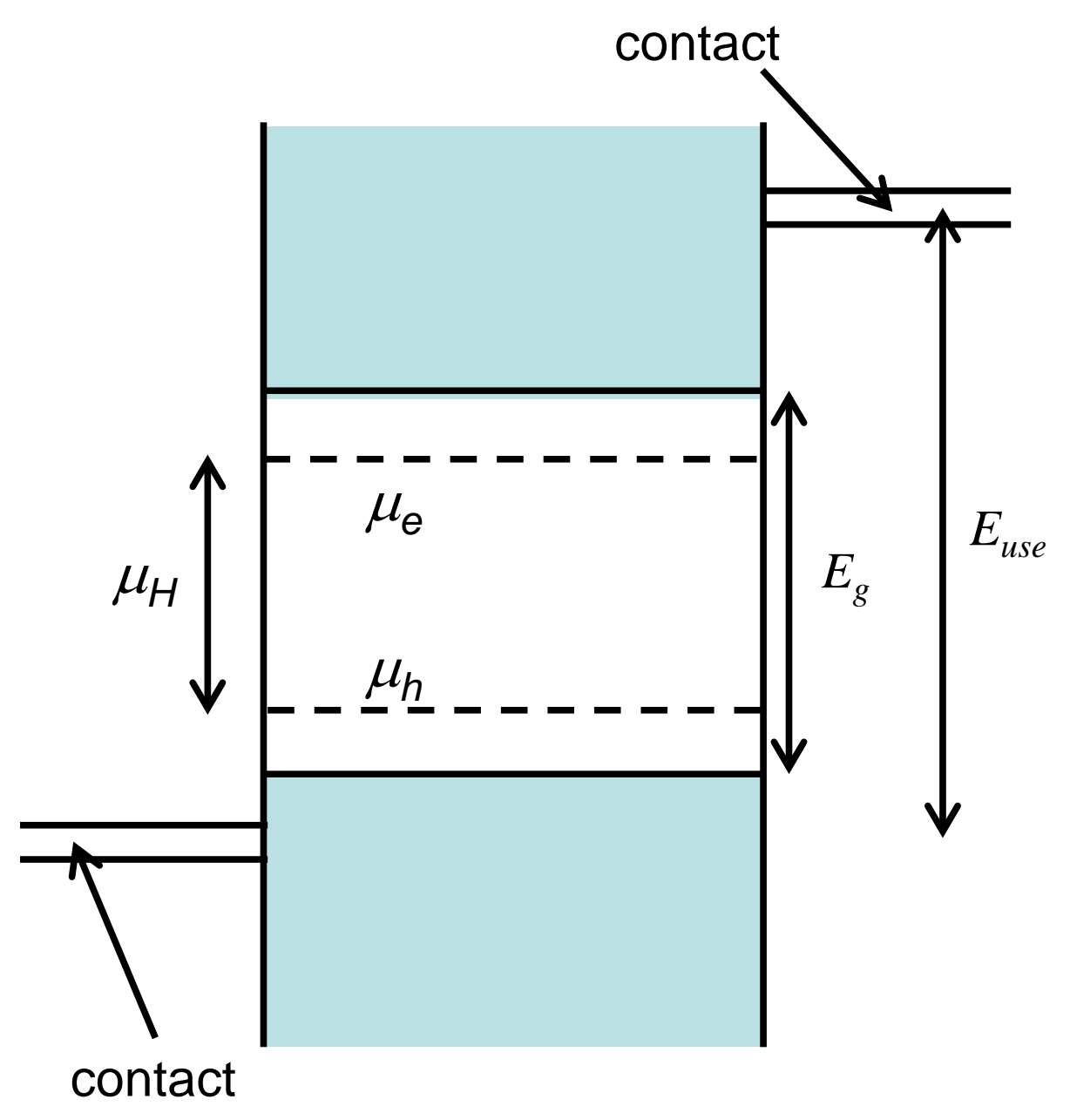

(a)

$$
T_{S}, \mu=0, \mathscr{\mathscr { C }}_{\text {in }} \quad T_{H}, \mu_{H} \leq 0, \mathscr{\mathscr { C }}_{\text {out }}
$$
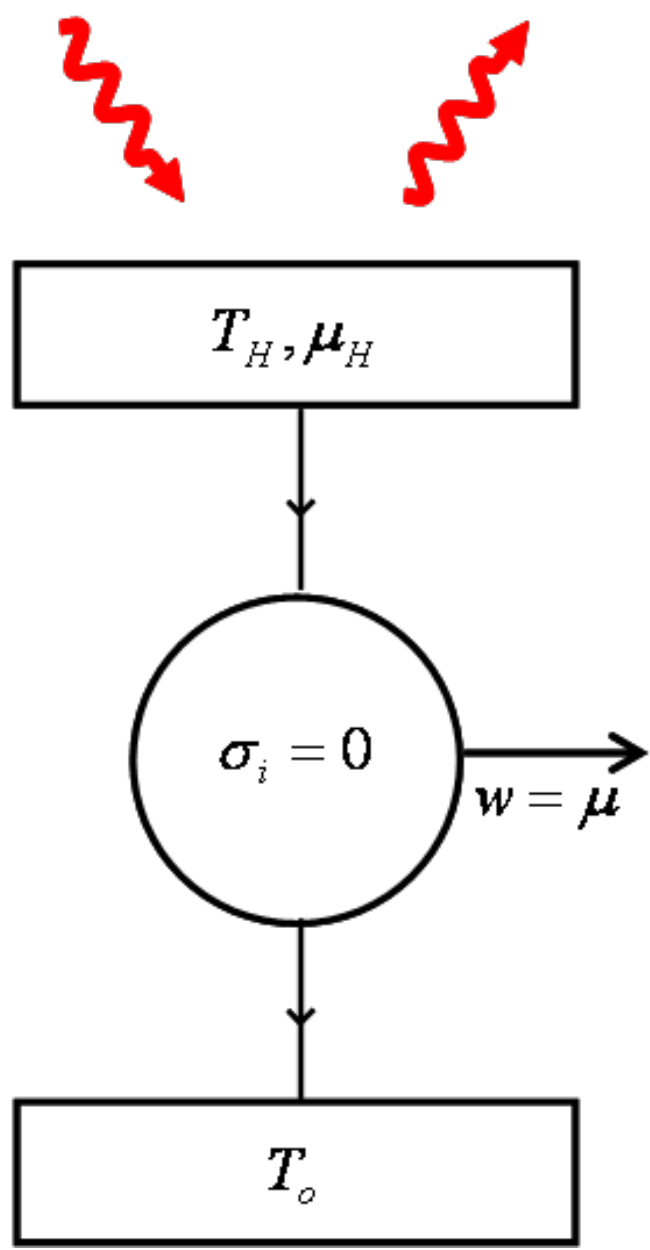

(b)

Fig. 9. The concept of hot carrier solar cell (a) based on selective contacts, and (b) as a more general "thermodynamic" converter. 

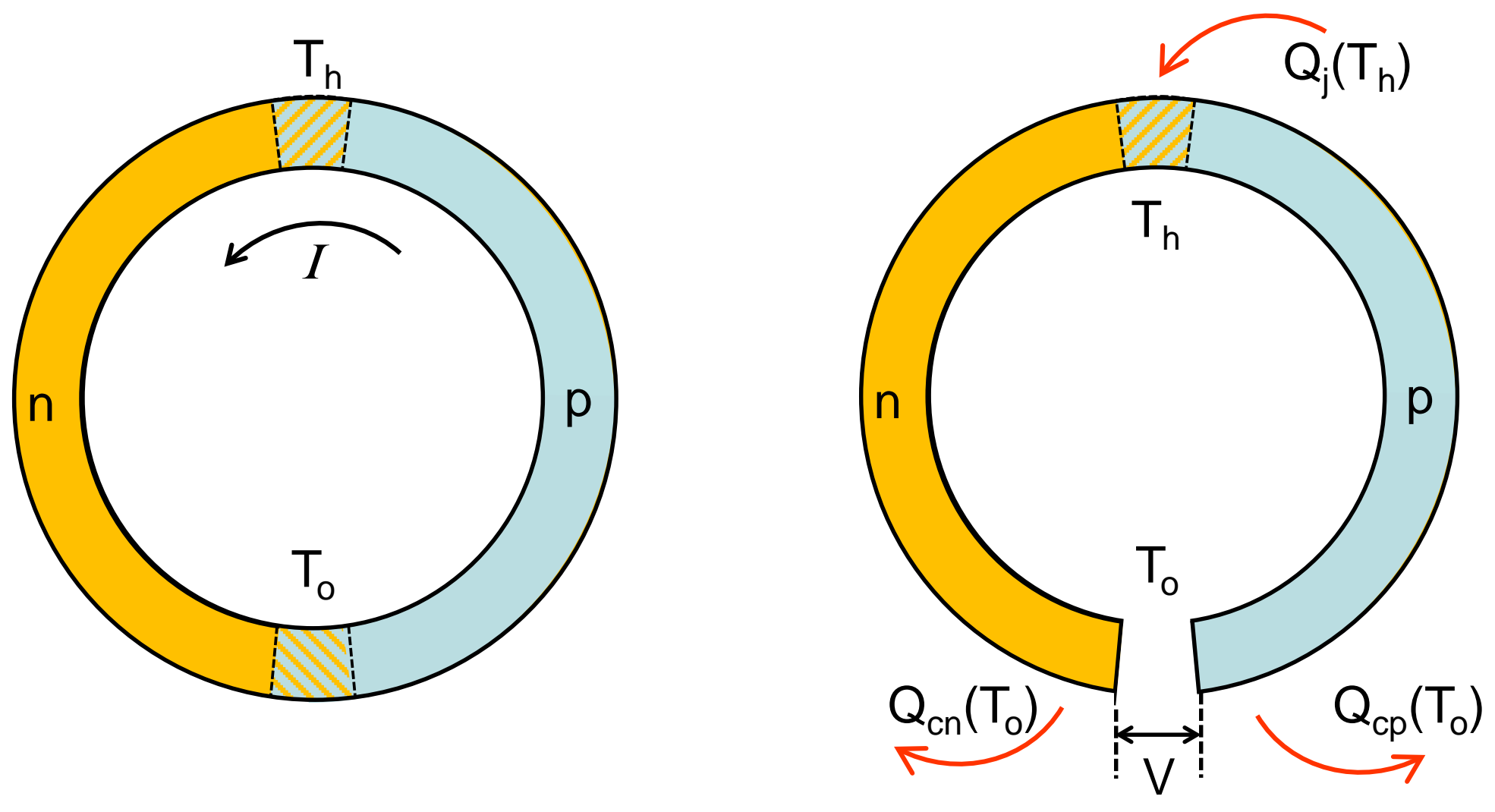

(a)

(b)

Fig. 10. Schematic diagrams of thermoelectric circuits producing current (a) and voltage (b). 


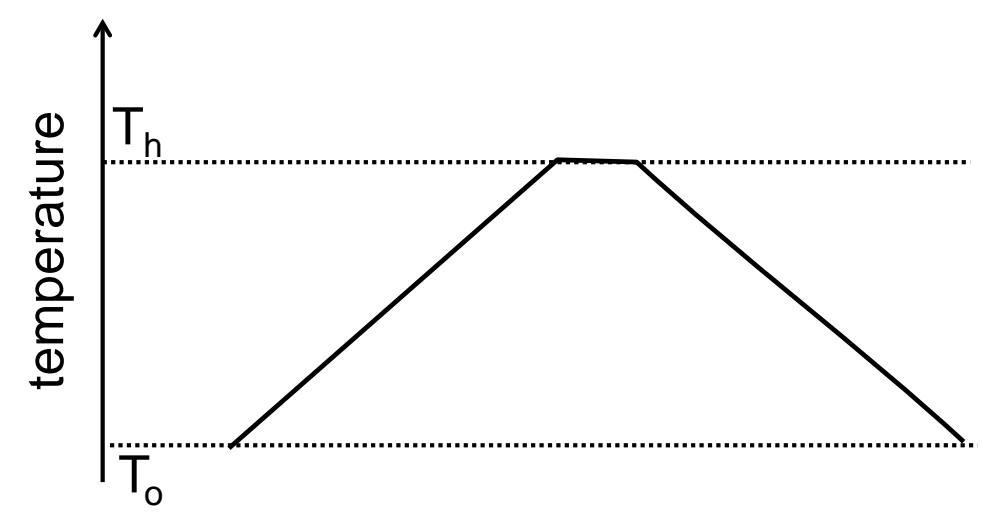

(a)

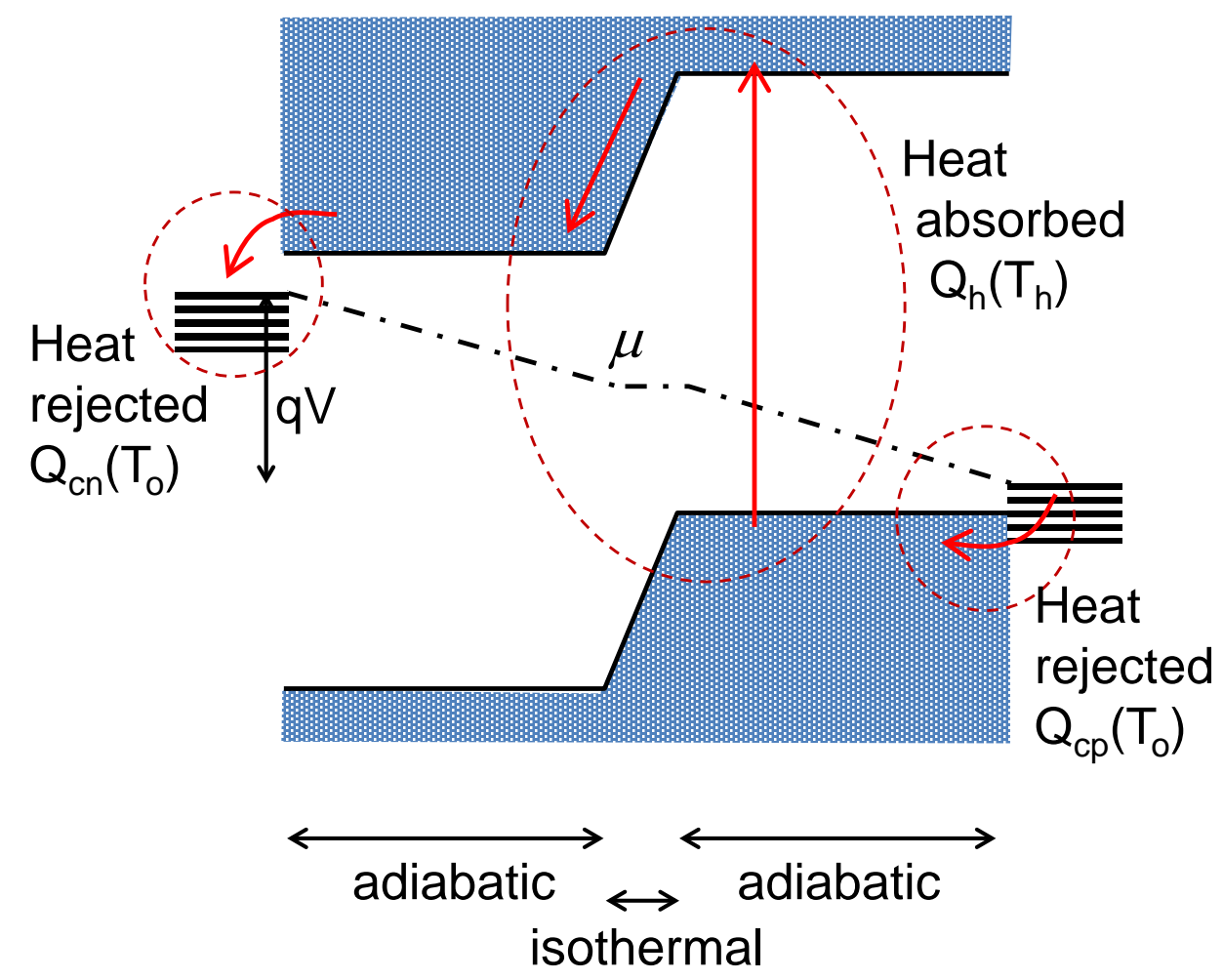

(b)

Fig. 11. The temperature profile (a) and band diagram (b) of a semiconductor thermoelectric voltage generator in Fig. 10b. 


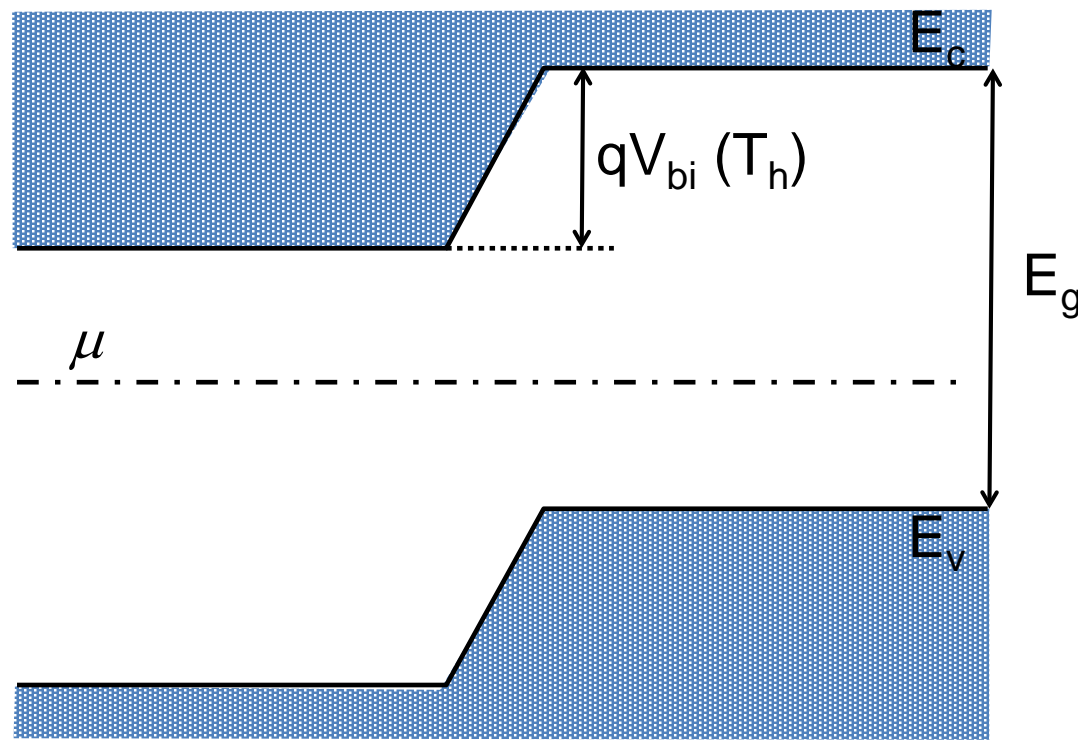

(a)

Fig. 12. Voltage generation in a conventional solar cell where hot carriers are created in equilibrium at a temperature $T_{h}(\mathrm{a})$, followed by thermalization and charge separation, giving rise to the splitting of quasi-Fermi levels / chemical potentials (b).

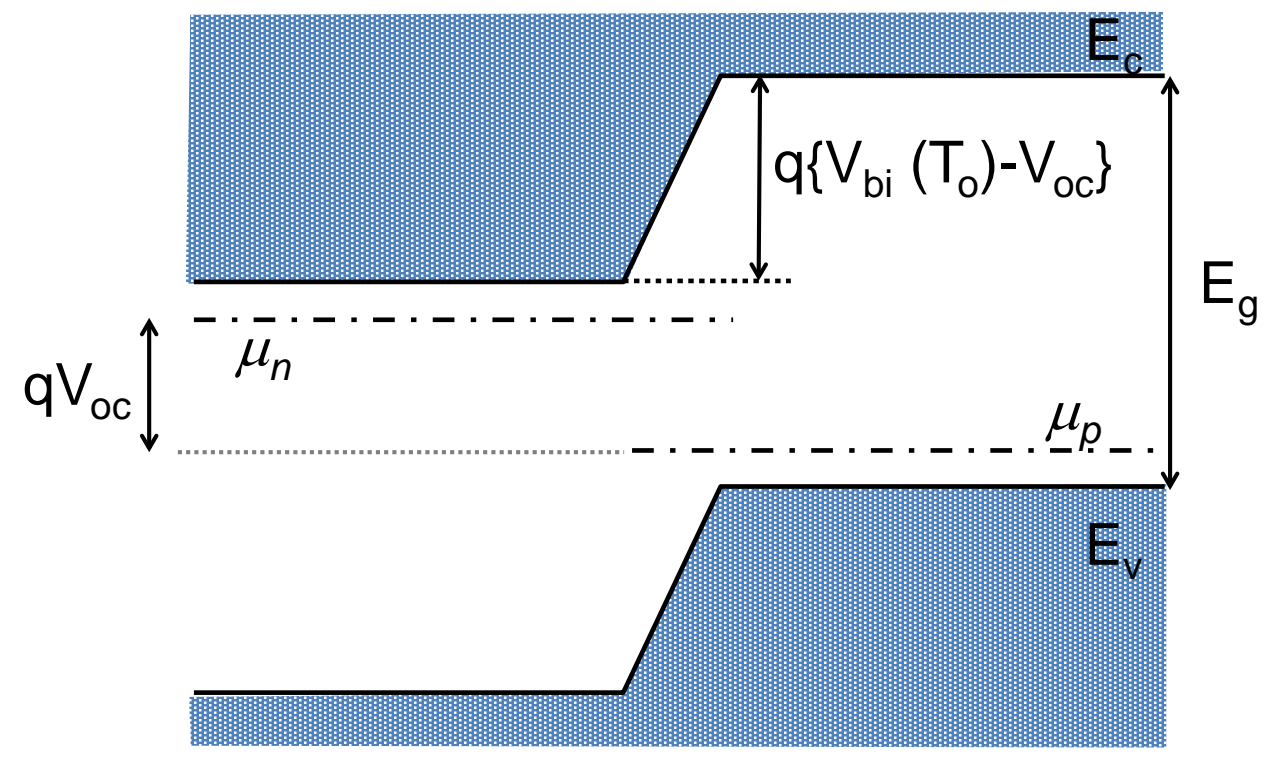

(b) 